\title{
Corela
}

Cognition, représentation, langage

18-2 | 2020

Vol. $18, n^{\circ} 2$

\section{Description sémantique des noms de doctrines et d'attitudes suffixés en -isme}

\section{Grigory Agabalian}

\section{(2) OpenEdition}

\section{Journals}

Édition électronique

URL : https://journals.openedition.org/corela/12672

DOI : $10.4000 /$ corela. 12672

ISSN : $1638-573 \mathrm{X}$

Éditeur

Cercle linguistique du Centre et de l'Ouest - CerLICO

\section{Référence électronique}

Grigory Agabalian, "Description sémantique des noms de doctrines et d'attitudes suffixés en -isme», Corela [En ligne], 18-2 | 2020, mis en ligne le 03 février 2021, consulté le 22 juillet 2021. URL : http:// journals.openedition.org/corela/12672; DOI : https://doi.org/10.4000/corela.12672

Ce document a été généré automatiquement le 22 juillet 2021.

\section{(c) (i) (2)(2)}

Corela - cognition, représentation, langage est mis à disposition selon les termes de la licence Creative Commons Attribution - Pas d'Utilisation Commerciale - Partage dans les Mêmes Conditions 4.0 International. 


\section{Description sémantique des noms de doctrines et d'attitudes suffixés en - isme}

Grigory Agabalian

\section{Introduction ${ }^{1}$}

1 Cet article s'intéresse à un sens construit par la suffixation en -isme et qu'on peut gloser comme une valorisation de ce que représente la base (Roché $2011: 72)$ :
(1) a. anarchisme 'valorisation de l'anarchie'
b. carriérisme 'valorisation de la carrière'
c. évolutionnisme 'valorisation de l'évolution'

2 Sur le plan référentiel, les ISMEs porteurs de ce sens valorisationnel sont principalement des noms de doctrines, de théories, de religions, de mouvements (artistiques, politiques, religieux) et certains noms d'attitudes.

3 Dans la première section, nous verrons que le sens valorisationnel des ISMEs n'est pas toujours glosable par 'valorisation de BASE'. D'une part parce que la base peut parfois représenter l'agent qui valorise - c'est-à-dire 'valorisation (de quelque chose) par BASE' et non plus 'valorisation de BASE (par quelqu'un)'. D'autre part, parce qu'il y a des écarts entre le sens construit, (purement morphologique) et les sens attestés (plus complexes et qu'on peut rencontrer dans les dictionnaires, les encyclopédies ou les discours). Dans tous les cas, nous appellerons ici «sens valorisationnel» un sens construit par la suffixation en -isme et qui comporte deux rôles sémantiques, ceux d'objet valorisé et d'agent qui valorise, qu'on peut instancier par la forme de l'ISME (par la base ou le radical).

4 Lorsqu'un ISME fait l'objet d'une interprétation valorisationnelle, cette interprétation semble être toujours opérée dans un contexte de rivalité qui oppose l'entité valorisée (une idée, une personne, une valeur...) à une autre entité. Ce phénomène a déjà été vérifié, sur corpus, dans Agabalian (2019b). Cet article s'attachera donc à se demander 
comment intégrer ce fait dans une description formalisée du sens valorisationnel des ISMEs. Ce sera l'objet de la section 2.

5 Un mot sur le corpus. Nous avons recueilli 1513 entrées lexicales différentes en -isme dans le Trésor de la langue française informatisé (TLFi) et dans le Petit Robert 2017² (PR17) réunis. Nous avons nettoyé le corpus en éliminant, notamment les entrées dont aucune définition ne restitue un sens valorisationnel - tel que défini plus haut - dans aucun des deux dictionnaires (analphabétisme, exorcisme, héroïsme, journalisme...), d'autant plus si elles ne sont pas analysables comme des formes construites (schisme, séisme...), celles qui étaient construites par préfixation (anticolonialisme, néolibéralisme...) ou par composition (marxisme-léninisme) et celles définies comme un comportement de valorisation du fait du sens de la base et non pas de la suffixation en -isme (chauvinisme, narcissisme...) ${ }^{3}$. Nous sommes parvenu à un corpus de 783 ISMEs construits par suffixation en -isme et porteurs d'un sens valorisationnel dans au moins une de leurs définitions ou dans le TLFi ou dans le PR17. Ce corpus n'a pas été constitué pour répondre à la question « quel est l'inventaire des ISMES valorisationnels en français? » - la délimitation discrète d'un tel inventaire est impossible. Ce corpus a été constitué pour répondre à la question "quand on attribue un sens valorisationnel à un ISME, comment représente-t-on ce sens? ». Nous avons dépouillé les définitions de ces 783 ISMEs, nous avons analysé leur structure formelle et nous les avons soumis à des tests sémantiques. Enfin, pour obtenir des informations sur l'histoire des doctrines, théories ou religions dénommées par un ISME, nous avons consulté des encyclopédies, des dictionnaires ou autres ouvrages spécialisés. Sauf indication contraire, les analyses qui suivent sont les résultats de ce travail.

\section{Le sens valorisationnel construit par la suffixation en -isme}

6 Nous partons du travail le plus approfondi à l'heure actuelle sur la suffixation en -isme, à savoir Roché (2011) qui identifie, parmi les différents sens que la suffixation en -isme peut construire, un sens que le morphologue qualifie d' « axiologique » et qui consiste en une valorisation de ce que représente la base $\mathrm{4}^{4}$. Cette valorisation se fait, d'après Roché, au nom du vrai, du bien, du beau ou de ce qui est important. Nous avons requalifié ce sens de « valorisationnel » et voici comment nous le décrivons :

(<résultat $>$ de la) $<$ valorisation $>$ de $<x>$ par $<y>$

7 Cette glose représente le sens construit des ISMES valorisationnels, c'est-à-dire qu'elle a pour seule fonction de convertir leur forme en sens. Elle est constituée de trois variables qui sont les produits de la suffixation en -isme: <valorisation $>,<\mathrm{x}>$ et $\langle\mathrm{y}>$. La quatrième variable, <résultat $>$, ne fait pas partie du sens construit car elle n'est pas le fait de la suffixation en -isme toutefois elle joue un rôle très important. C'est par elle que nous commencerons.

\section{$1.1<$ résultat>}

8 Cette variable renvoie à la forme référentielle à travers laquelle la valorisation se manifeste dans le monde. Le plus souvent il s'agit d'une doctrine. Mais on trouve aussi des théories, des mouvements, des religions, des idéologies, des dogmes ainsi que des 
attitudes, des tendances, des comportements, parfois des régimes, des styles, des méthodes, des procédés, des modes de vie. Les noms qui peuvent instancier cette variable sont, théoriquement, des hyperonymes pour les ISMEs et réussissent, de ce fait, un test de Hiérarchie-Être (Bever \& Rosenbaum, 1971) :

(2) a. L'évolutionnisme est une théorie

b. Le carriérisme est une attitude

c. Le nominalisme est une doctrine

9 L'instanciation de <résultat> doit se faire à partir d'un corpus (lexicographique, encyclopédique ou autre) car elle n'est pas prédictible. Nous avons extrait, manuellement, de notre corpus les noms qui sont les incluants (Rey-Debove 1971: 230-243) de toutes les définitions de chacun des 783 ISmEs et qui, de ce fait, sont aussi susceptibles d'être leurs hyperonymes. Nous avons confié le comptage de ces noms au logiciel $\mathrm{TXM}^{5}$. Voici le résultat pour les principaux noms (à partir de 20 occurrences minimum) :

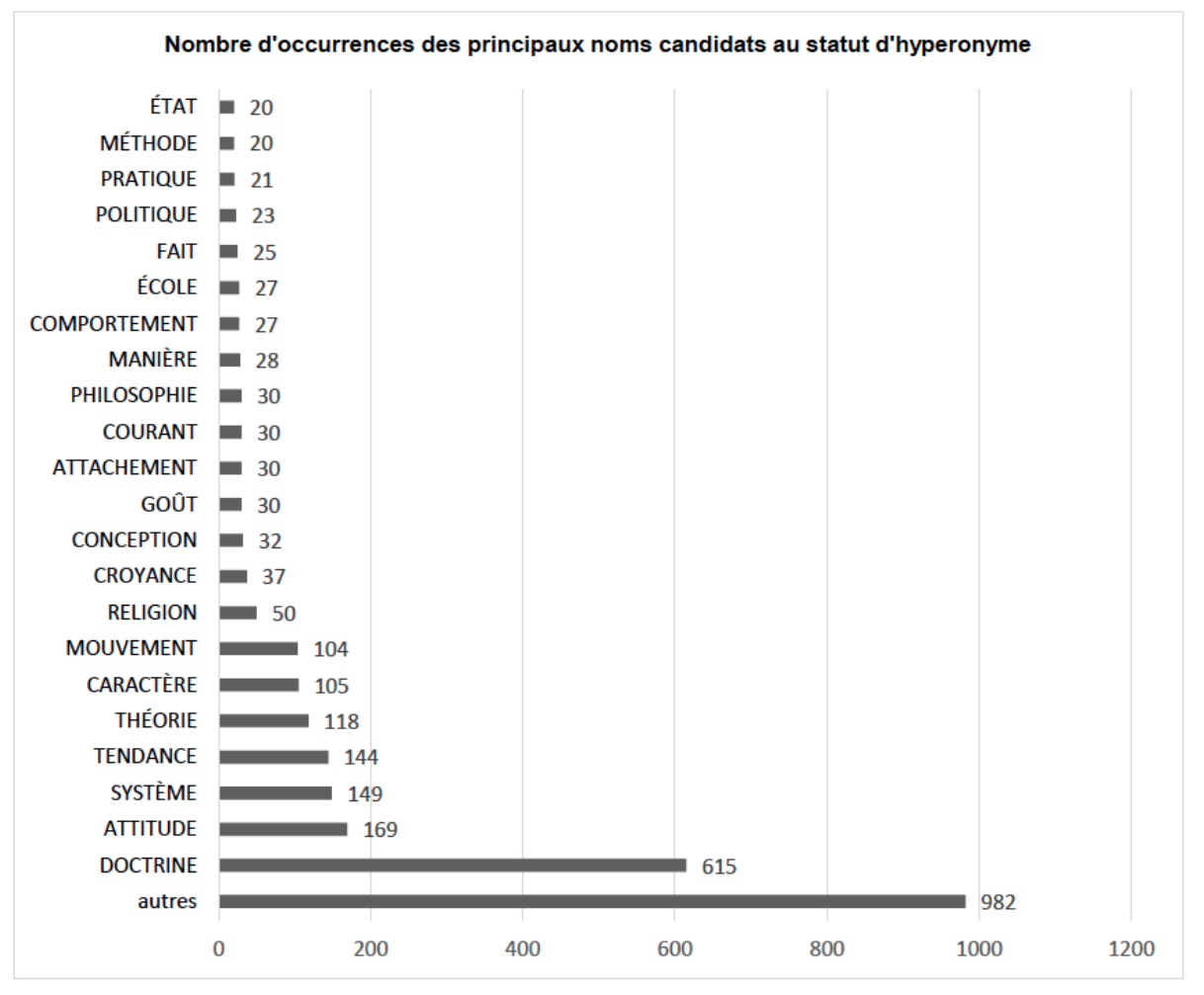

Quelques précisions :

1) La catégorie « autres » regroupe 350 noms affichant tous moins de 20 occurrences.

2) Sur la méthode : pour tout ISME retenu, les incluants de toutes les définitions ont été comptabilisés, même ceux des définitions qui ne correspondent pas à une interprétation valorisationnelle. Par exemple, instrumentalisme compte trois définitions dont seulement deux restituent une interprétation valorisationnelle. Le nom qui sert d'incluant dans ces deux définitions a évidemment été comptabilisé (doctrine, deux occurrences) mais l'incluant de la définition non valorisationnelle (caractère) a également été comptabilisé :

(3) Instrumentalisme :

1. Doctrine pragmatique de l'Américain J. Dewey, suivant laquelle [...]. (TLFi)

2. Caractère instrumental, utilitaire (d'un objet de connaissance). (TLFi)

3. Doctrine pragmatique suivant laquelle [...]. (PR17) 
Cela signifie que des incluants participant à la représentation de sens non valorisationnels (comme caractère) ont été « embarqués » dans la comptabilisation avec les incluants qui participent à la représentation d'un sens valorisationnel (comme doctrine). Cette situation concerne essentiellement comportement, manière, fait, caractère, état, méthode et pratique; très peu attitude, tendance, politique, système, conception et mouvement; pas du tout attachement, goût, doctrine, croyance, école (au sens de 'courant de pensée'), courant, philosophie, religion, théorie. Lorsqu'une définition comporte plusieurs incluants, nous les avons tous comptabilisés (sensationnalisme: "goût, recherche $d u$ sensationnel " (TLFi)). S'ils ont moins de 20 occurrences, eux et leurs occurrences ont été inclus dans la catégorie « autres ».

14 3) Tous les noms du graphique ne sont pas des hyperonymes. Il faut en effet vérifier si ces noms ont un pouvoir classifiant pour les ISMEs. Par exemple dans :

(4) Formalisme (PR17) :

1. Attachement scrupuleux aux formes extérieures, aux règles de la vie sociale,

religieuse, etc.

2. Doctrine qui soutient que les vérités scientifiques sont purement formelles [...].

Doctrine est un hyperonyme pour formalisme car il réussit le test de Hiérarchie-Être qu'on trouve chez différents auteurs, notamment Rey-Debove (1971 : 230) ou Kleiber \& Tamba (1990:17) : Le formalisme est une doctrine. En revanche, attachement ne réussit pas ce test ('Le formalisme est un attachement) et ne peut donc pas fonctionner comme hyperonyme. Il en va de même pour goût (sensationnalisme: "goût, recherche $d u$ sensationnel »(TLFi)). Il s'ensuit qu'attachement et goût ne peuvent pas instancier la variable <résultat $>$. Ils instancient plutôt la variable <valorisation> que nous verrons en 1.2 .

16 Voyons maintenant comment se répartissent les ISMES du corpus à partir du sens de ces noms hyperonymes. Sur le plan référentiel, nous pouvons distinguer quatre types de référents :

17 1) Les noms de systèmes d'idées : doctrine, religion, théorie, philosophie, conception, croyance $^{6}$ (au sens de 'croyance religieuse, philosophique ou théorique'), système (au sens de 'système d'idées'), dans certains cas école (au sens de 'courant de pensée'). Il s'agit d'ISMEs qui dénomment des constructions théoriques cohérentes, plus ou moins complexes et soutenant des valeurs et/ou des idées.

18 2) Les noms de projets idéologiques : mouvement, courant, dans certains cas école (au sens de 'courant de pensée'). Il s'agit d'ISMEs qui dénomment des activités d'influence (plus ou moins bien définies) menées par divers groupes de pression (plus ou moins conscients d'eux-mêmes et parfois sous l'autorité d'un leader) pour faire pénétrer dans un groupe un changement de normes, de méthodes, de pratiques politiques, économiques, sociales, sexuelles, alimentaires, esthétiques, etc. Ainsi, ce qui distingue, par exemple, féminisme défini comme doctrine et féminisme défini comme mouvement, c'est qu'en tant que doctrine féminisme dénomme une construction théorique, alors qu'en tant que mouvement, il dénomme un ensemble d'activités d'influence entrepris dans une société par un groupe de pression pour y faire pénétrer un changement de normes. Même si les noms de projets idéologiques peuvent aussi dénommer très facilement des contenus idéologiques ou des collectifs humains, ce qui les caractérise le plus c'est cet aspect dynamique et prospectif (des activités orientées vers un futur idéal). Être adossé à une construction théorique cohérente, stable ou consensuelle a 
beaucoup moins d'importance que le fait de simplement regarder vers une nouvelle (et meilleure selon les partisans) façon de faire, de penser ou de vivre.

3) Les noms de manières (d'être ou de faire) situées : système (au sens de 'système de fonctionnement' ou 'système d'organisation'), méthode, pratique, politique. Il s'agit d'ISMEs qui dénomment des manières d'être ou de faire dans un domaine d'activité spécifique, principalement la politique, l'art, la science, la religion et l'économie.

4) Les noms de manières (d'être ou de faire) libres: attitude, tendance, comportement, marginalement caractère, manière, état et fait. Il s'agit d'ISMES qui dénomment des manières d'être ou de faire qu'on peut rattacher à n'importe quel domaine d'activité ou à aucun domaine en particulier.

21 Ces noms peuvent se cumuler pour un même ISME, soit dans des définitions différentes soit dans la même définition :

(5) Psychologisme (TLFi) :

1. Tendance à accorder une trop grande place à la psychologie [...] ;

2. en partic., doctrine selon laquelle la psychologie, les faits psychiques sont le

fondement de toutes les sciences humaines.

(6) Activisme : Doctrine ou méthode d'action [...]. (TLFi)

Cette situation soulève la question de la polysémie des ISMEs et tout particulièrement de la polysémie doctrine/attitude (matérialisme, rationalisme, réalisme, traditionalisme, etc.). Pour le cas des définitions à plusieurs têtes (comme activisme), on peut aussi se demander si le recours au concept de facette (Cruse, 1996) ne serait pas pertinent ${ }^{7}$.

Sur le plan sémantique, la diversité des noms qui instancient cette variable <résultat> met en évidence le fait que les ISMES valorisationnels ne constituent pas une classe sémantique mais ont une distribution sémantique multiclasse :

1) Les noms d'idéalités mentales/pragmatiques (Flaux \& Stosic, 2015) pour ceux qui sont catégorisés comme doctrine, théorie, mouvement, religion, croyance, système (de pensée), école, courant, philosophie, conception dans le graphique mais on peut ajouter idéologie, dogme, culte, opinion, etc. présents dans notre corpus mais avec moins de 20 occurrences. Cette classe rassemble les noms de systèmes d'idées et les noms de projets idéologiques.

2) Les noms intensifs (Flaux \& Van de Velde, 2000) et, plus précisément, les noms de qualités psychologiques et/ou d'attitudes (Anscombre, 1995). La différence entre qualité psychologique et attitude n'est pas toujours évidente mais il s'agit de tous les ISMES catégorisés comme attitude, tendance, comportement et parfois caractère et état. Cette seconde classe renvoie, grosso modo, aux noms de manières libres.

26 3) Il reste un troisième groupe d'ISMEs que nous n'avons pas réussi à classer pour le moment, ce sont ceux catégorisés par méthode, politique, pratique, système (de fonctionnement, d'organisation) dans le graphique mais aussi par style, mode de vie, régime, organisation, mode de gouvernement, etc. Ils sont tous des noms de manières situées. Nous serions tenté de les classer comme noms d'idéalités praxématiques (Flaux \& Stosic, 2015) mais c'est un point à vérifier.

Enfin, précisons que la variable <résultat> ne fait pas partie des éléments construits par la suffixation en -isme, de sorte qu'on peut se contenter de définir les ISMEs seulement avec le prédicat de valorisation :

(7) Autonomisme : revendication d'autonomie. (PR17)

(8) Sensationnalisme : goût, recherche du sensationnel (TLFi) 
enre de définition, où le prédicat de <valorisation> apparaît sans son <résultat>, est tout à fait cohérent avec l'histoire des suffixes -isme et -iste. En effet, ces derniers viennent du grec ancien où ils servaient à former des noms d'actions (pour -isme) et d'agents (pour -iste), autrement dit des sens processifs ${ }^{8}$.

\section{$1.2<$ valorisation>} $(<y>)$. Comme pour <résultat>, son instanciation doit se faire à partir d'un corpus car ce n'est pas le genre d'information qu'on peut prédire à partir de la forme de l'ISME. Nous avons réussi à dégager deux types d'instanciations pour <valorisation> au niveau des sens attestés ${ }^{9}$ :

1) Les valorisations locutoires : affirmer, réclamer, revendiquer, demander, prôner, etc. Le sens des ISMES concernés est littéralement en forme d'acte d'énonciation rapporté.

(9) Immanentisme : Doctrine qui affirme l'immanence de Dieu [...]. (PR17)

Sens construit: '(<résultat : doctrines qui) <valorisation : affirme $><\mathrm{x}$ : l'immanences'

(10) Altermondialisme : Mouvement qui [...] réclame d'autres modèles économiques, sociaux, écologiques et culturels (PR17)

Sens construit : '(<résultat : mouvement > qui) <valorisation : réclame ><x : un autre monde>'

(11) Naturisme : Doctrine selon laquelle l'adoration des forces de la nature est à l'origine des religions. (TLFi)

Sens construit : '(<résultat : doctrine $>$ ) <valorisation : (assertif - selon laquelle) $><\mathrm{x}$ :

la natures'

Pour naturisme, on peut rétablir l'acte assertif sous-jacent à selon lequel au moyen d'un verbe (affirmer) ou indiquer que <valorisation> est instancié par un acte assertif que les limites des données du corpus ne permettent pas de spécifier. Par ailleurs, on peut voir que l'instanciation de $<x>$ est incomplète d'une telle manière que la glose en devient inintelligible (voir 1.3.1).

2) Les valorisations non-locutoires : privilégier, aimer, avoir confiance en, avoir le goût de, rechercher, désirer, être attaché à, etc. Contrairement au cas précédent, la valorisation n'est pas en forme d'acte d'énonciation rapporté.

(12) Passéisme : attachement excessif au passé [...]. (TLFi)

Sens construit : '(<résultat $>$ ) <valorisation : attachement $><x$ : au passé $>$

(13) Affairisme: tendance à ne s'occuper que d'affaires particulièrement lucratives

[...] (PR17)

Sens construit: '(<résultat : tendance> à) <valorisation : ne s'occuper que $><\mathrm{x}$ : d'affaires>'

(14) Stylisme: tendance à travailler beaucoup son style, à privilégier parfois excessivement la forme par rapport au fond ${ }^{10}$. (TLFi)

Sens construit: '(<résultat : tendance> à) <valorisation : travailler beaucoup; privilégier $><x$ : le style>'

Dans la glose, les mots grammaticaux (de, à, que, ce, qui, le...) peuvent être modifiés, supprimés, placés dans ou hors des chevrons pour rendre la glose intelligible et lisible. De même l'instanciation de <valorisation> peut se faire au moyen d'un nom, d'un verbe ou d'un syntagme, ça n'a pas d'importance. 

valorisation modale: la valorisation qu'ils signifient est effectivement une modalisation épistémique ou boulique/déontique positive. Sur le plan de la classification sémantique, ils sont très souvent des noms d'idéalités mentales/ pragmatiques et ont donc, presque toujours, des noms comme doctrine, théorie, mouvement, religion comme hyperonymes pour instancier leur variable <résultat>. Ils réussissent tous un test qui consiste à les traiter comme des énonciateurs rapportés grâce aux syntagmes prépositionnels selon le $\mathrm{N}$ et/ou pour le $\mathrm{N}$ moyennant, parfois, l'ajout d'un auxiliaire modal déontique :

(15) Selon l'immanentisme, $p$

Pour l'altermondialisme, on doit $\mathrm{p}$

Selon le naturisme, $\mathrm{p}$

Les ISMES pour lesquels la valorisation n'est pas en forme d'acte d'énonciation sont des ISMES à valorisation attitudinale : la valorisation qu'ils signifient est une manière d'être ou de faire observable à travers tout ce qui constitue l'attitude de quelqu'un (ce qu'il dit, comment il le dit, sa manière de partager l'espace et les biens avec les autres, ses réactions physiques, etc.). Sur le plan de la classification sémantique, ils sont tous des noms de qualités psychologiques et/ou d'attitudes (peut-être aussi d'idéalités praxématiques si l'appartenance à cette classe s'avère pertinente, voir 1.1). Leur variable <résultat> est donc presque toujours instanciée par des hyperonymes comme attitude, tendance ou comportement ${ }^{11}$. Ils échouent au test précédent :

(16) *(Selon / Pour) l'affairisme, p

*(Selon / Pour) le passéisme, p

*(Selon / Pour) le stylisme, $\mathrm{p}$

La situation de polysémie doctrine/attitude, évoquée en 1.1, pour la variable $<$ résultat> concerne aussi la variable <valorisation>. Ainsi, lorsqu'un ISME signifie à la fois une doctrine et une attitude (qui va avec cette doctrine), sa valorisation est modale en tant que doctrine et attitudinale en tant qu'attitude. Voir l'exemple de psychologisme en (5).

2) Nous avons dit plus haut que, comme <résultat>, la variable <valorisation> doit être instanciée à partir d'un corpus car cette instanciation ne peut pas être prédite à partir de la forme de l'ISME. Ajoutons maintenant qu'à l'inverse de <résultat>, la variable <valorisation> est produite par la suffixation en -isme, c'est-à-dire que c'est grâce à sa forme construite par suffixation en -isme qu'un ISME est apte à signifier la valorisation de quelque chose.

3) Précisons aussi que <valorisation> ne signifie pas nécessairement trouver que quelque chose est bien. En effet, la valorisation peut tout à fait porter sur quelque chose qu'on trouve indésirable. C'est le cas pour défaitisme, qui signifie '<valorisation : croire à ; être certain de $><x$ : la défaite>'. De sorte que <valorisation $>$ doit être compris comme un métaprédicat qui n'est pas du tout limité au sens appréciatif positif du lexème valorisation. Nous paraphrasons là Roché (2011 : 73-74).

40 4) Enfin, la valorisation peut être excessive (cérémonialisme: "attachement étroit aux règles d'un culte » (TLFi)), ce qui rend toujours péjoratif l'ISME concerné. 
41 Pour résumer et d'après notre corpus, on peut dire que le sens valorisationnel s'organise de la manière suivante au niveau des sens attestés (« ov » signifie «objet valorisé ») :

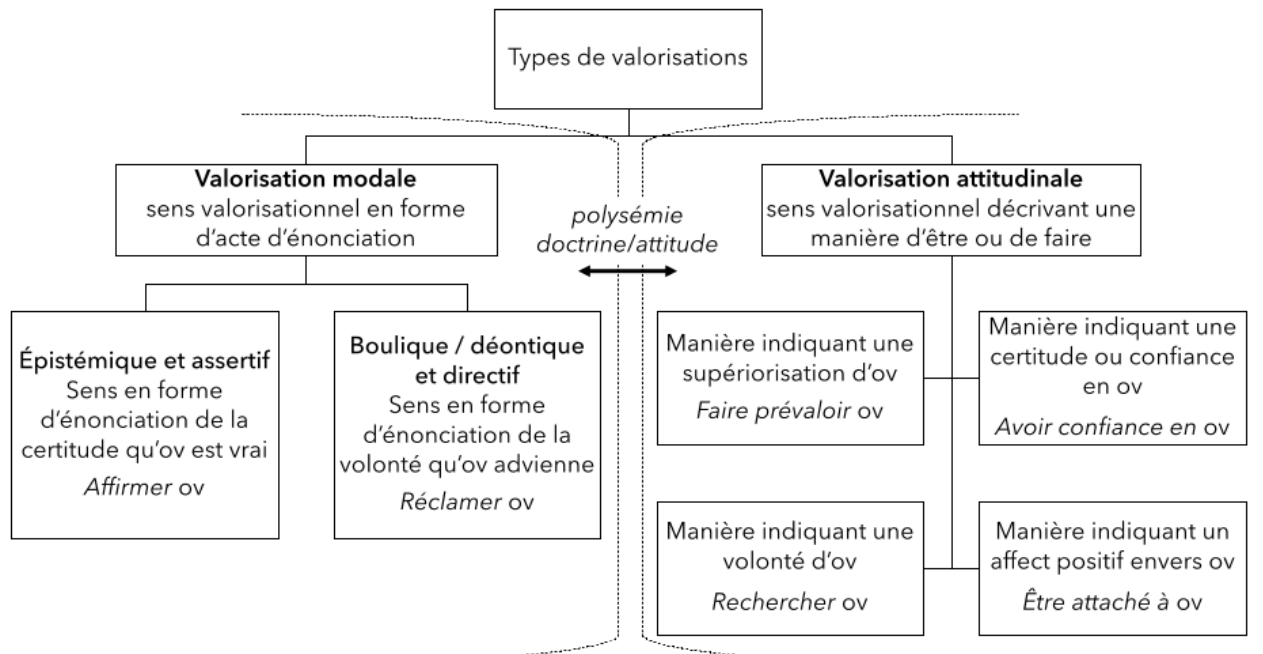

Schéma 1. Organisation de l'instanciation de la variable < valorisation>

\section{$1.3<x>$ et $<y>$}

Nous avons vu que les variables < résultat> et <valorisation> devaient être instanciées par des unités prélevées depuis un corpus. Les variables $<x>$ et $<y>$, elles, doivent être instanciées par des unités prélevées depuis la forme de l'ISME et rien d'autre que sa forme. Autrement dit, la suffixation en -isme construit trois variables (<valorisation>, $<\mathrm{x}>$ et $<\mathrm{y}>$ ) et la forme de l'ISME fournit l'instanciation de deux d'entre elles $(<\mathrm{x}>$ ou $<\mathrm{y}>)$. La méthode évidente pour l'instanciation de ces deux variables est la suivante :

1) On retire le suffixe -isme de la forme de l'ISME; il reste alors une forme radicale.

2) À travers la matérialité de cette forme radicale, on reconnaît un lexème (éventuellement tronqué), une unité non lexicale ou un syntagme français, autrement dit on reconnaît une unité qui peut prétendre être la base de l'ISME ${ }^{12}$.

3) On détermine le rôle actanciel de cette unité dans la glose en consultant les sens attestés de l'ISME. Si elle remplit le rôle de l'objet valorisé, alors elle instancie $<\mathrm{x}>$; la glose simplifiée que nous utiliserons sera '<valorisation $>$ de $<x>$ ' :

(17) a. évolution évolutionnisme '<valorisation $>$ de $<\mathrm{x}$ : l'évolution>'

b. naturisme naturisme '<valorisation $>\mathrm{de}<\mathrm{x}$ : la nature $>$ '

Si elle remplit le rôle de l'agent qui valorise, alors elle instancie $<y>$; la glose simplifiée que nous utiliserons sera ' $<\mathrm{x}$ : ce que $><\mathrm{y}><$ valorise $>$ ' 13 :

(18) a. protestant protestantisme ' $<\mathrm{x}$ : ce que $><\mathrm{y}:$ le protestant $><$ valorise'

b. végane véganisme ' $<\mathrm{x}$ : ce que $><\mathrm{y}$ : le végane $><$ valorise $>$ '

Cette méthode simple ne fonctionne pas toujours. Voyons donc les difficultés rencontrées. 


\subsubsection{Instanciation de $<x>$ : écarts avec l'objet valorisé dans les sens attestés}

lobjet qui est in fine valorisé dans les sens attestés. On peut citer l'exemple de sionisme dont le sens construit est rigoureusement '<valorisation $>$ de $<\mathrm{x}$ : Sion>'. Or, dans les sens attestés de ce lexème, l'objet valorisé est quelque chose comme un foyer national juif en Israël. De la même manière neptunisme a rigoureusement pour sens construit '<valorisation> de <x : Neptune>'. Or, là aussi, lorsqu'on consulte les dictionnaires on se rend compte que le sens attesté est celui d'une doctrine qui affirme que les formations rocheuses sont dues à l'eau. Par conséquent, l'objet qui est in fine valorisé dans le sens attesté n'est pas Neptune mais l'eau.

3) L'unité qui instancie < $x>$ est un hyperonyme de l'objet qui est in fine valorisé dans les sens attestés. L'exemple typique est racisme. Son sens construit est rigoureusement '<valorisation> de <x : la race>'. Or, là encore, l'observation des sens attestés montre des écarts. D'abord, dans l'un de ses sens attestés race est complété par un prédicat ('<valorisation> de $<\mathrm{x}$ : les races> (sont hiérarchisées)') mais surtout dans un autre sens attesté, c'est l'un de ses hyponymes qui est valorisé : '<valorisation> de $<\mathrm{x}$ : la race> (blanche)'. Sexisme et spécisme suivent le même modèle.

\subsubsection{Instanciation de $<x>$ : rattachement du radical à une unité française qui serait la base} régler. Ceux que nous allons aborder maintenant concernent strictement la formation des ISMEs mais ont des incidences majeures sur ce qui nous intéresse (la description sémantique).

1) La forme radicale est celle d'un adjectif : quelle est la catégorie de la base ?

Lorsqu'on soustrait /ism/ à égalitarisme on obtient la forme radicale /egalitar/, laquelle est celle de l'adjectif égalitaire et pas celle du nom égalité. Ainsi, la base d'égalitarisme devrait être l'adjectif égalitaire. Or, d'après Roché (2011 : 74-75, 82-83), la base des ISMES valorisationnels (« axiologiques » dans sa terminologie) est un nom, c'est-à-dire qu'ils 
sont construits en relation avec un nom même si leur radical est celui d'un adjectif. C'est, du moins, ce qui est le plus cohérent sémantiquement. Toujours d'après lui, ce sont des raisons phonologiques et surtout des phénomènes d'effet de série qui font que le nom base peut se matérialiser en empruntant le thème dérivationnel de l'un des adjectifs de sa famille dérivationnelle ${ }^{14}$. Ainsi égalitarisme est bien dérivé d'égalité (pas d'égalitaire) et, pour notre description sémantique, cela signifie qu'il a pour sens construit '<valorisation> de <x : l'égalité>'. De même que nationalisme est dérivé de nation (pas de national) et signifie '<valorisation> de <x : la nation>'. Nous suivons Roché sur ce point. Mais deux observations s'imposent.

La première est qu'il reste des cas où la base est ambiguë. Par exemple modernisme en tant que nom de qualité/attitude (pas en tant que nom de doctrine) pourrait aussi bien être dérivé du nom modernité tronqué (attitude de '<valorisation> de $<\mathrm{x}$ : la modernité') que de l'adjectif moderne et signifier 'qualité de ce qui est moderne', auquel cas il faut déterminer si modernisme et modernité forment une paire minimale de noms de qualités - question que se pose justement Roché (2011: 108-109). D'autre part, il existe des paires minimales du type naturisme / naturalisme qui pourraient donner tort à l'analyse Roché selon laquelle la base est toujours un nom. Le morphologue constate que ces paires sont souvent des ISMEs formés dans des domaines d'activités différents ou que l'un d'eux est emprunté à une autre langue (Roché $2011: 78)^{15}$.

La seconde observation est que, de manière beaucoup plus générale, la difficulté à "décider » d'une base pour les noms en -isme et en -iste a fait partie des arguments pour démontrer le caractère pas toujours opérant d'une description morphologique canonique ${ }^{16}$ puisque cette description conduit à prendre des décisions qui paraissent parfois arbitraires. Face à ce type de problème, on a proposé un modèle paradigmatique qui veut que les membres de plusieurs familles morphologiques (nation, national, nationaliste (A), nationaliste ( $\mathrm{N})$, nationalisme... est une famille; loi, légal, légaliste (A), légaliste $(\mathrm{N})$, légalisme... est une autre famille) instancient les cellules morphologiques (X, Xal, Xaliste, Xalisme...) d'un réseau de relations sémantiques (un objet et la propriété qui lui est relative, un objet et l'agent qui le valorise, un objet et sa valorisation...). Chacun des membres d'une famille est ainsi connecté aux autres membres de sa famille et la construction d'un lexème ne repose plus sur l'identification vaille que vaille d'une et une seule base mais sur le fait que ce lexème instancie une cellule qui «veut dire quelque chose " pour tous les autres membres de sa famille et qu'en même temps cette organisation en réseau se répète pour d'autres familles ${ }^{17}$. La description canonique n'est pas pour autant bannie de ce modèle. Elle s'applique seulement à certaines relations du réseau. Celles qui nous intéressent sont, en l'occurrence, celles entre l'objet valorisé, sa valorisation et l'agent de la valorisation.

\section{2) La forme radicale n'est pas française : à quelle unité française la rattacher ?}

Pour répondre à ce problème, on trouve d'abord une distinction intuitive :

1) Le locuteur français assimilera la forme radicale à une unité française (notamment grâce à une redondance consonantique) : par exemple le radical latin /popyl/ dans populisme sera assimilé à peuple; ce qui signifie que peuple peut être la base de populisme et donc, pour ce qui concerne notre description sémantique, que le sens construit '<valorisation $><\mathrm{x}$ : du peuple>' est recevable $\mathrm{si}<\mathrm{x}>$ prétend pouvoir être la base (ce qui est toujours le cas). 
60 2) Le locuteur français n'assimilera pas la forme radicale à une unité française : par exemple il n'assimilera pas /edon/ à plaisir pour hédonisme ni /ate/ à pas de dieu pour athéisme; ce qui signifie que plaisir et pas de dieu ne peuvent pas être les bases d'hédonisme et d'athéisme et donc, pour ce qui concerne notre description sémantique, que les sens construits '<valorisation $><x$ : du plaisir $>$ ' et '<valorisation $>$ de $<x$ : pas de dieu>' ne sont pas recevables $s i<\mathrm{x}>$ prétend pouvoir être la base.

Pour le cas 2, le seul à poser vraiment problème, il y a deux traitements qui s'opposent à propos du rôle du locuteur :

i) Si on part du principe qu'identifier l'objet valorisé sur le plan sémantique équivaut à identifier la base sur le plan de la formation, alors il faut considérer qu'on a identifié cette base dès lors qu'on a traduit la forme radicale en français et qu'on a reconnu dans cette traduction l'objet valorisé dans le sens. Ces radicaux grecs ou latins seraient donc des thèmes ${ }^{18}$ supplétifs savants de lexèmes, d'unités non lexicales ou de syntagmes français servant de bases pour certaines opérations morphologiques (essentiellement certaines suffixations et la composition néoclassique) ${ }^{19}$.

63 Cette solution peut poser deux problèmes. D'abord, elle peut ignorer les connaissances linguistiques des locuteurs: soit elle fait comme si tout le monde était érudit en étymologie, soit elle part du principe que ce ne sont pas les connaissances linguistiques des locuteurs qui décident de comment un lexème est construit, qu'il y a une réalité des unités formant un lexème qui n'a pas à dépendre de la manière dont un locuteur comprend la forme de ce lexème. Ensuite - second problème - même si l'analyse par thèmes supplétifs s'appuie sur la recherche de distributions complémentaires (pour éviter la première critique), elle donne parfois l'impression d'ignorer les écueils soulevés par la traduction. Par exemple $\dot{\eta} \delta o v \eta ́$ se traduit par plaisir, agrément ou jouissance en français dans le Bailly (en ligne). Lequel de ces lexèmes retenir comme base d'hédonisme ${ }^{20}$ ?

64 ii) Si on part du principe que le locuteur francophone "moyen » ne rattachera jamais spontanément les radicaux des ISMES concernés à des unités françaises, on peut opter pour une autre analyse formelle qui soutient que la base de ces ISMEs est le nom d'adepte : hédonisme ' $<\mathrm{x}$ : ce que $>\mathrm{y}$ : l'hédoniste $><$ valorise $>$ ' et athéisme ' $<\mathrm{x}$ : ce que $><\mathrm{y}$ : l'athée $><$ valorise>' (comme $<\mathrm{x}>,<\mathrm{y}>$ prétend toujours pouvoir être la base). Cette solution, qui consiste à se rabattre sur le nom d'adepte pour fournir une base à la construction d'un ISME, semble a priori toujours possible car un ISME valorisationnel a toujours un nom d'adepte : qu'il soit en -iste (hédoniste), en -ique (cynique pour cynisme), en -ien (chrétien pour christianisme) ou autre (cathare pour catharisme).

65 Cette seconde solution pose clairement que le locuteur francophone "moyen ", entité générique et purement virtuelle, est le facteur de contrôle par excellence pour décrire la formation du lexème. Il y a deux problèmes avec cette approche. D'abord, il faudrait faire le portrait prototypique de ce locuteur moyen. Mel'čuk, Clas \& Polguère (1995: 43-44), par exemple, le décrivent comme ayant un niveau d'instruction supérieur et exerçant un métier intellectuel (avocat, journaliste...), âgé d'environ 50 ans et parlant le français «standard» de France. Que faire des autres francophones? Ensuite - second problème - nous sommes réticent à l'idée qu'un nom d'adepte en -iste puisse constituer la base d'un ISME valorisationnel et réciproquement. Comme le dit bien Roché (2011: 74), les deux formes, -isme et -iste, se motivent toujours l'une l'autre (par exemple esclavagisme $\longleftrightarrow$ esclavagiste (N/A)) et cette motivation réciproque est très utile pour les 
locuteurs lorsque le lien avec une base commune s'est opacifié, n'a jamais existé en français ou est méconnu (par ex. : pessimiste et pessimisme par rapport à pessimus 'le pire' en latin) mais, pour autant, elles ne constituent pas des bases de construction l'une pour l'autre. Une telle description nous ramène à la solution de la morphologie paradigmatique évoquée plus haut.

D'autres approches existent évidemment. Ainsi, Baeskow (2004) - en morphologie «morpheme-based» - choisit de créer, pour l'anglais, des entrées lexicales indépendantes pour ces radicaux supplétifs savants. Par exemple nihil- (de nihilism) n'est pas considéré comme une forme supplétive de nothing mais dispose de sa propre entrée dans le lexique anglais (en tant qu'unité d'origine non-germanique et de forme toujours liée).

\subsubsection{Instanciation de <x> : présence d'un préfixe}

Lorsqu'un préfixe est présent (néolibéralisme, anticolonialisme) la question de l'identification d'une base n'est pas un impératif dans une description paradigmatique mais dans une description canonique il faut déterminer si l'ISME a été formé par suffixation en -isme ( néolibéral? ou néolibéralité? néolibéralisme), par préfixation (libéralisme néolibéralisme) voire par parasynthèse (libéral néolibéralisme).

Sans chercher à trancher cette question, disons que très souvent le sens peut nous indiquer laquelle des deux unités (le préfixe ou le suffixe) produit un ajout sémantique. Et c'est ce qui importe pour notre description sémantique. Comparons préromantisme et préadamisme. Le premier dénomme une période artistique qui se situe avant (pré-) le romantisme, par conséquent le préfixe pré- ou son sens 'avant' ne font pas partie de ce qui est valorisé. Ils sont ajoutés à romantisme qui, de ce fait, peut prétendre être la base d'une préfixation :

(19) romantisme préromantisme 'période avant le romantisme'

Préadamisme est différent. Il ne dénomme pas du tout la période avant l'adamisme. Il dénomme une doctrine qui affirme qu'un premier homme aurait existé avant (pré-) Adam. Autrement dit, pré- et son sens 'avant' font partie de ce qui est valorisé et c'est le suffixe -isme qui a été ajouté à cet objet pour exprimer sa valorisation. Ce qui, dans certaines théories morphologiques (voir plus haut), peut laisser croire que préadam- est un radical supplétif pour une base de suffixation:

(20) avant Adam (forme préadam-) préadamisme '<valorisation> de (l'existence

d'un premier homme) <x : avant Adam>'

Conformément à ce qui a été dit en introduction sur la constitution du corpus, ont été retenus seuls les ISMEs dont le sens est analysable comme le résultat d'un ajout de -isme alors que le préfixe ou son sens font partie de l'objet valorisé (comme préadamisme). Du reste, le préfixe peut aussi apparaître tel quel dans l'instanciation de $<\mathrm{x}>$, notamment quand il s'agit des préfixes $a$ - (anarchisme '<valorisation> de <x : l'anarchie>'), in(indépendantisme '<valorisation> de $<\mathrm{x}:$ l' indépendance'>) ou dé- (déconstructivisme ' $<$ valorisation $>$ de $<x$ : la déconstruction $>$ ').

Trois choses sont à noter :

72 1) À côté des ISMEs à préfixe, on en trouve quelques-uns qui paraissent construits par composition : anarchosyndicalisme, anglo-catholicisme, judéo-christianisme, judéo-hellénisme, logicopositivisme, marxisme-léninisme, national-socialisme, etc. Il est très difficile de 
déterminer si leur sens est le résultat d'une suffixation en -isme à partir d'une base composée ou syntagmatique (qui exprimerait l'objet valorisé ou l'agent qui valorise) ou si leur sens est le résultat d'une composition, auquel cas le suffixe -isme serait porté par le deuxième composant seulement ou par les deux composants mais en étant tronqué sur le premier. À partir d'indices lexicographiques et encyclopédiques faibles, nous avons considéré que leur sens pouvait être analysé comme le résultat d'une suffixation en -isme et les avons donc inclus dans notre corpus de 783 ISMEs, à l'exception de marxisme-léninisme qui est clairement un composé.

2) On trouve également des ISMEs se terminant par -centrisme (égocentrisme, ethnocentrisme, etc.) et pour lesquels il convient de se demander quel est le statut de centr-. Le segment -centrisme semble circuler en bloc pour s'attacher à des radicaux souvent supplétifs. Pour autant, les ISMES concernés ne sont pas construits par composition (ethnocentrisme n'est pas le résultat d'ethnie + centrisme). En fait, il semblerait que -centr- soit une sorte de co-suffixe qui spécifie l'interprétation de -isme. Il indique que l'objet valorisé ne l'est pas n'importe comment, il l'est en faisant de lui un centre (vers lequel tout converge, depuis lequel tout part ou autour duquel tout tourne). Ces ISMEs ont donc été inclus dans notre corpus.

74 3) Le cas de la préfixation par anti-, avec sa valeur adversative, est le plus important quantitativement. Les ISMES concernés sont presque toujours construits par préfixation en anti- à partir d'un ISME base puisqu'ils dénomment l'attitude, la doctrine ou le mouvement qui est hostile à cet ISME :

(21) Anticolonialisme : Opposition au colonialisme [...]. (PR17)

Une telle analyse contrevient à celle qui est habituellement appliquée à la préfixation en anti- adversatif puisqu'il est établi que cette dernière construit des adjectifs (Heyna 2008 : $\$ 18$; Hathout 2011 : 311 entre autres) :

(22) limace $_{\mathrm{N}} \quad$ antilimace $_{\underline{\underline{A}}}$ 'qui repousse / protège contre les limaces'

Or, les données sémantiques de notre corpus indiquent que les constructions suivantes décrivent mal le sens attesté des anti-ISMEs à sens adversatif :

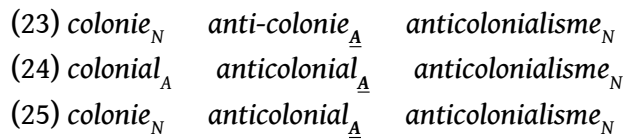

Alors que la construction suivante coïncide mieux avec le sens :

(26) colonialisme $e_{N}$ anticolonialisme $e_{N}$

78 En effet, le sens attesté d'anticolonialisme laisse penser que c'est anti- qui ajoute sémantiquement quelque chose à colonialisme et non -isme qui ajoute sémantiquement quelque chose à anticolonial ou à anti-colonie ${ }^{21}$. On comprendra plus loin pourquoi antiadversatif peut directement produire le nom «adverse » à partir d'un ISME (section 2). Pour l'heure, disons que les anti-ISMEs de nos sources lexicographiques ont un sens qui montre qu'ils ne sont pas analysables comme étant formés par suffixation en -isme et, de ce fait, ont été exclus des 783 ISMES.

\subsubsection{Instanciation de $<y>$ : les noms propres d'animés}

Si la base est un nom propre d'animé (NPan), elle peut être aussi bien l'agent qui valorise $(<y>)$ que l'objet valorisé $(<x>)$ :

1) NPan agent ('<x: ce que $><y$ : (NPan) $><$ valorise>') : 
(27) a. Marx marxisme '<x : ce que $><\mathrm{y}$ : Marx $><$ valorise $>$ ' (doctrine de Marx)

b. Calvin calvinisme ' $<\mathrm{x}:$ ce que $><\mathrm{y}:$ Calvin $><$ valorise $>$ ' (doctrine de Calvin)

2) NPan objet ('<valorisation $>$ de $<x$ : (NPan) $>$ ') :

(28) a. Bonaparte bonapartisme '<valorisation $>\mathrm{de}<\mathrm{x}$ : Bonaparte>' (attachement à

Bonaparte)

b. Molière moliérisme '<valorisation $>\mathrm{de}<\mathrm{x}$ : Molière>' (admiration pour Molière)

En 1), NPan est une source intellectuelle et en 2) il peut être une idole (28) ou un représentant (métaphorique, métonymique, stéréotypique...) de l'objet qui est, in fine, valorisé dans le sens attesté. C'est le cas de neptunisme déjà cité plus haut.

Nous ferons trois observations sur les ISMES valorisationnels avec un NPan comme base :

1) Il est rare qu'un même NPan occupe les deux rôles actanciels. Parmi les exemples que nous avons trouvés dans de notre corpus on peut citer deux luciférianisme dérivés de deux Lucifer homonymes et voltairianisme :

(29) a. Lucifer (le démon) luciférianisme '<valorisation> de <x : Lucifer>' (culte de

Lucifer)

b. Lucifer (l'évêque) luciférianisme '<x : ce que> <y : Lucifer (de Cagliari)>

<valorise>' (doctrine de Lucifer de Cagliari - un évêque sarde du IVe siècle)

(30) Voltaire voltairianisme

'<valorisation $>$ de $<\mathrm{x}$ : Voltaire>' (goût pour les idées de Voltaire)

'<x : ce que > <y: Voltaire > <valorise>' (philosophie de Voltaire)

Les exemples sont contestables. Dans un cas, il s'agit de deux NPan homonymes, dans l'autre la base pourrait être le nom d'adepte voltairien pour le sens agentif. Nous verrons ce cas en 1.3.5.

2) Nous n'avons pas effectué de recherche détaillée pour déterminer le facteur qui pourrait expliquer pourquoi le NPan instancie l'un ou l'autre rôle actanciel. Toutefois, à première vue, il semblerait que cela dépende des données encyclopédiques. Les NPan faisant référence à des animés surnaturels ou à des êtres divinisés sont presque systématiquement dans le rôle d'objet valorisé, en tant qu'idoles (quelques fois en tant que représentants). Les NPan faisant référence à des artistes sont très souvent aussi des idoles. Les NPan de philosophes, de savants et d'hommes de religion semblent presque toujours être dans le rôle d'agent et, plus exactement, celui de source intellectuelle. On trouve aussi, parfois, des NPan fictifs dans ce même rôle de source intellectuelle (orphisme, pantagruélisme). Enfin, les NPan faisant référence à des hommes politiques ou à des chefs militaires semblent être les plus fluctuants.

3) Les ISMEs dérivés d'un nom propre de femme sont quasi-inexistants dans les dictionnaires que nous avons consultés. Si on ne tient compte ni de la différence nom propre / nom commun ni de la différence entre sens valorisationnel et non valorisationnel, les figures féminines que l'on trouve sont : la mère (maternalisme), la Vierge Marie (marianisme), la prostituée (putanisme, cocottisme), la lesbienne (saphisme, lesbianisme), la femme qui conteste la domination masculine (féminisme), l'actrice (actricisme) et la femme insatisfaite affectivement (bovarysme). Il n'y a aucun NPan féminin dans le rôle de source intellectuelle dans notre corpus mais quelques rares cas existent en dehors (par ex. : arendtisme ${ }^{22}$.

\subsubsection{Instanciation de $<y>$ : radicaux dérivés d'un nom propre d'animé}

Certaines formes radicales correspondent à des noms d'adeptes dérivés d'un NPan, tout particulièrement des noms d'adeptes en -ien: /egeljan/ pour hégélianisme, /kartezjan/ 
pour cartésianisme, /kristjan/ pour christianisme, etc. Le problème est qu'on peut se demander si la base est le nom propre ou le nom d'adepte :

(31) a. hégélien ${ }_{\mathrm{N}}$ hégélianisme '<x : ce que><y : l'hégélien><valorise>'

b. Hegel hégélianisme ' $<\mathrm{x}$ : ce que $><\mathrm{y}$ : Hegel $><$ valorise>'

Comme avec les préfixés (néolibéralisme, anticolonialisme), ce problème d'identification d'une base n'est pas un frein pour procéder à une description paradigmatique. Dans une description canonique, en revanche, la question se pose même si elle n'a pas d'incidence majeure pour notre description sémantique. Si l'on suit Roché (2007 : 56), les noms de religions, de sectes ou d'hérésies formés avec un radical en -ian-auraient suivi le modèle fourni par christianisme. D'où arianisme, luthérianisme, marianisme, messianisme, sabellianisme, etc. Autrement dit, christianisme (l'un des ISMEs les plus anciens, emprunté au grec) aurait agi comme un mot leader pour imposer un modèle de formation aux nouveaux ISMES relatifs au domaine référentiel de la religion: l'important étant moins la conformité formelle avec la base que la reproduction de la rime /janism/ pour maintenir l'unité formelle de la série. Or, ce segment -ian- est également très présent dans le domaine de la philosophie. D’après Roché, ce modèle de formation en -ianisme n'a pas fait pression seulement sur la formation des ISMES religieux mais aussi sur celle des ISMEs philosophiques si l'on admet qu'il a existé, dans l'esprit des locuteurs, une porosité entre doctrine religieuse et doctrine philosophique. Ainsi, pour Roché (2007: 53-55), la base d'hégélianisme et de cartésianisme est respectivement Hegel et Descartes, et non pas l'adepte en -ien. Lignon, Namer \& Villoing (2014 : 1817-1818) confirment, dans l'ensemble, l'analyse de Roché.

\subsection{La variable $<b>$}

Nous avons vu que l'instanciation de $<\mathrm{x}>$ pose différents problèmes : écarts avec l'objet valorisé dans le sens attesté et instanciations contestables à partir de la forme à partir du moment où $\langle x>$ prétend pouvoir être la base (surtout avec les radicaux étrangers). Pour que ces deux difficultés formelles ne nous empêchent pas d'atteindre la description du sens valorisationnel des ISMES nous avons décidé de passer par une variable $<\mathrm{b}>$.

\subsection{1 $<\mathrm{b}>$ pour remplacer $<\mathrm{x}>$}

La variable $<b>$ est instanciée par des noms, des syntagmes ou des propositions qui représentent l'objet valorisé (comme $<\mathrm{X}>$ ) mais qui sont prélevés depuis un corpus (et non depuis la forme de l'ISME) et, surtout, qui ne prétendent pas pouvoir être la base. Voici des exemples pour illustrer la différence entre $\langle x>$ et $\langle b>$ :

Sens d'abolitionnisme avec <x> : '<valorisation> de <x : l'abolition>'

$<\mathrm{x}>$ est instancié par une unité prélevée depuis la forme d'abolitionnisme - et rien d'autre que sa forme - et qui peut constituer la base d'abolitionnisme.

\section{Sens d'abolitionnisme avec <b> : '<valorisation > de < : l'abolition de l'esclavage>'}

$<b>$ est instancié par un syntagme prélevé depuis un corpus lexicographique. L'instanciation $\mathrm{de}\langle\mathrm{b}\rangle$, contrairement à celle $\mathrm{de}\langle\mathrm{x}\rangle \mathrm{ou}\langle\mathrm{y}\rangle$, ne prétend pas pouvoir être la base de l'ISME. cette variable prétend seulement être l'objet valorisé en tant que cet 
objet valorisé est une information sémantique toujours présente dans le sens attesté d'un ISME, quelle que soit la base de ce dernier.

Sens de marxisme avec $<\mathrm{x}>$ : '<valorisation $>$ de $<\mathrm{x}>$ par $<\mathrm{y}$ : Marx $>$ ' $(<\mathrm{x}:$ ce que $><\mathrm{y}$ : Marx > <valorise >')

$<\mathrm{X}>$ reste non instancié car il doit être instancié par une unité prélevée depuis la forme de marxisme et qui peut constituer la base de marxisme. Or, cette unité remplit, ici, le rôle de l'agent qui valorise. Par conséquent, l'objet valorisé $(\langle x>)$ reste inconnu (ce que est une simple formule de remplissage pour rendre la glose lisible).

Sens de marxisme avec $<\mathrm{b}>$ : '<valorisation $>$ de $<\mathrm{b}:$ le capitalisme est une aliénation> par $<\mathbf{y}$ : Marx>>

$<b>$ est instancié par une proposition recueillie depuis un corpus et qui ne prétend pas pouvoir être la base de marxisme.

Sens d'athéisme avec instanciation de $<\mathrm{x}>$ : '<valorisation> de $<\mathrm{x}$ : pas de dieu> par $<y>$

Sens d'athéisme avec instanciation de <y> : '<x : ce que><y : l'athée> <valorise>'

Sens d'athéisme avec $<\mathrm{b}>$ : '<valorisation> de $<\mathrm{b}$ : l'inexistence de Dieu>'

On voit qu'avec la variable $<b>$ les questions soulevées en 1.3.2 sont contournées : il n'y a plus besoin de savoir si la base d'athéisme est le syntagme pas de dieu matérialisé sous la forme radicale supplétive athé- ou si on ferait mieux de considérer que la base est plutôt le nom d'adepte athée. Il en va de même pour les ISMEs dont la forme radicale est celle d'un adjectif (nous avions donné l'exemple d'égalitarisme en 1.3.2). Ces questions sont contournées mais pas résolues car pour le morphologue, il reste à déterminer comment sont construits ces lexèmes.

Sens de neptunisme avec $<\mathrm{x}>$ : '<valorisation $>$ de $<\mathrm{x}$ : Neptune $>$ '

Sens de neptunisme avec $<\mathrm{b}>$ : '<valorisation $>$ de $<\mathrm{b}$ : la formation des roches est due à l'eau>'

6 Là aussi, nous voyons la différence entre l'instanciation de $\langle\mathrm{x}\rangle$ et celle de $\langle\mathrm{b}\rangle$ et les différences pour la description du sens. L'instanciation de $\langle x>$ ne représente que symboliquement l'objet valorisé dans les sens attestés. On obtient cet objet valorisé en passant de $<\mathrm{x}>\mathrm{à}<\mathrm{b}>$.

\subsection{2 $<\mathrm{b}>$ n'est pas sans rapport avec $<\mathrm{x}>$ ou $<\mathrm{y}>$}

107 Comme nous l'avons dit, $<\mathrm{b}>$, contrairement à $<\mathrm{x}>\mathrm{ou}<\mathrm{y}>$, ne prétend pas être la base de l'ISME mais, d'un autre côté, il n'est jamais sans rapport avec eux. Voici quelques exemples de liens qu'on peut rencontrer dans les corpus de sens attestés des ISMEs (la plupart ont déjà été évoqués tout au long de notre analyse) :

\begin{tabular}{|l|l|}
\hline Relations $\mathrm{de}<\mathrm{b}>\mathrm{avec}<\mathrm{x}>$ et $<\mathrm{y}>$ & Exemple \\
\hline & passéisme $:<\mathrm{x}:$ le passé $>;<\mathrm{b}:$ le passé $>$ \\
$1 .<\mathrm{b}>=<\mathrm{x}>$ (traduit ou non) & athéisme $:<\mathrm{x}:$ pas de dieu $;<\mathrm{b}:$ pas de dieu $>$ \\
\hline
\end{tabular}




\begin{tabular}{|c|c|}
\hline 2. $\langle\mathrm{b}\rangle=\langle\mathrm{x}\rangle$ (traduit ou non) + arg. & 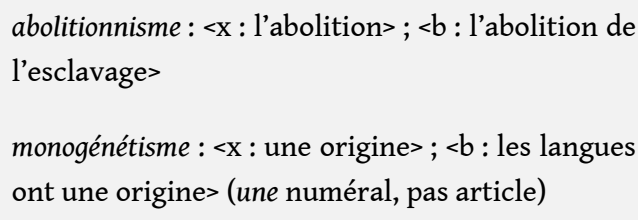 \\
\hline 3. $<b>=<x>$ (traduit ou non) + préd. & $\begin{array}{l}\text { racisme: }<\mathrm{x}: \text { la race }>\text {; }<\mathrm{b}: \text { les races sont } \\
\text { hiérarchisées }> \\
\text { hédonisme : <x : le plaisir }>\text {; }<\mathrm{b}: \text { le plaisir est le but } \\
\text { de l'existence }>\end{array}$ \\
\hline 4. $<\mathrm{b}>=$ synonyme de $<\mathrm{x}>$ & $\begin{array}{l}\text { stylisme: }<\mathrm{x} \text { : le style }>\text {; }<\mathrm{b}: \text { la forme }>\text { (par rapport } \\
\text { au fond) }\end{array}$ \\
\hline 5. $\langle\mathrm{b}>=$ périphrase de $<\mathrm{X}>$ & anarchisme : <x : l'anarchie $>$; $<\mathrm{b}:$ l'absence d'État $>$ \\
\hline 6. $\langle\mathrm{b}>=$ un hyponyme de $<\mathrm{x}>$ & racisme $:<\mathrm{x}$ : la race $>;<\mathrm{b}:$ la race blanche $>$ \\
\hline 7. $<b>=$ ce dont $<x>$ est le symbole & sionisme : $<\mathrm{x}$ : Sion $>;<\mathrm{b}:$ un État juif en Israël $>$ \\
\hline $\begin{array}{l}\text { 8. }\langle\mathrm{b}>=\text { les conditions, les moyens, } \\
\text { conséquences, les buts de la } \\
\text { valorisation de }<x>\end{array}$ & $\begin{array}{l}\text { écologisme: valorisation de }<\mathrm{x} \text { : l'écologie }>\text {; }<\mathrm{b} 1 \text { : } \\
\text { la décroissance; b2: la préservation des } \\
\text { ressources naturelles }>\end{array}$ \\
\hline 9. $<b>=$ les idées de $<y>$ & $\begin{array}{l}\text { chiisme: <y: chiite> ; <b: la succession de } \\
\text { Mahomet revient à Ali> }\end{array}$ \\
\hline
\end{tabular}

Tableau. Principaux liens entre $<x>$ et $<b>$ ou entre $<y>$ et $<b>$

Ces relations peuvent évidemment se combiner entre elles. Ce tableau n'est qu'illustratif. L'établissement de la plupart de ces relations a pour objectif de permettre de maintenir un lien entre $<b>$ et la forme de l'ISME, de montrer que $<b>$ est certes très variable mais reste sous le contrôle de la forme (c'est-à-dire de $\langle x>$ ou de $\langle y>$, que leur prétention au statut de base soit validée ou non). Le contrôle de la forme sur les instanciations de $<b>$ n'est pas de puissance égale dans toutes les relations. Sans être vraiment précis, disons que plus on descend dans le tableau, plus le lien entre le sens et la forme de l'ISME se distend et donc moins le contrôle du premier par la seconde se ressent. Les $<b>$ des huitième et neuvième types en particulier ne relèvent presque plus du cadre de cette description. 


\subsubsection{La variabilité de $<b>$ face à l'invariabilité de $\langle x\rangle$ et $<y>$}

109 L'instanciation de $<b>$ met en évidence le fait que les objets valorisés au niveau des sens attestés peuvent fortement varier alors que $<\mathrm{x}>\mathrm{ou}<\mathrm{y}>$ restent les mêmes.

110 1) $<b>$ peut varier thématiquement lorsqu'il y a une coupure contextuelle entre les différents objets valorisés de telle sorte que ces objets relèvent de contextes sociohistoriques ou intellectuels totalement déconnectés. Par exemple :

(32) a. naturisme : '<valorisation $>$ de $<\mathrm{x}$ : la nature>'

b. naturisme: '<valorisation> de

$<\mathrm{b} 1$ : un mode de vie en harmonie avec la nature ;

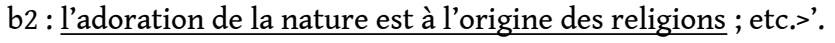

111 La variation thématique repose sur une coupure contextuelle qui laisse penser qu'il ne s'agit pas d'une "récupération-réinterprétation » du même lexème mais d'une même forme en -isme qui a été formée deux fois dans deux contextes sans rapport par deux groupes de locuteurs qui s'ignorent l'un l'autre. Il s'ensuit qu'aucune relation de polysémie régulière classique (métonymie, extension, métaphore...) n'est capable d'expliquer le passage d'un sens à l'autre car aucun des deux sens ne dépend d'une quelconque manière de l'autre.

2) Quand il n'y a pas de coupure contextuelle, $<b>$ peut varier :

113 - rhétoriquement:

(33) a. féminisme: '<valorisation $>$ de $<x$ : la femme>'

b. féminisme: '<valorisation> de

$<\mathrm{b} 1$ : l'émancipation des femmes ;

b2 : la domination des femmes sur les hommes ; etc.>

114 La variation rhétorique caractérise deux instanciations qui ont deux objectifs argumentatifs opposés pour un même ISME: l'une veut, par exemple, qu'on adhère à l'idée que l'ISME concerné dénomme quelque chose de bien, l'autre qu'on adhère à l'idée que le même ISME dénomme quelque chose de mal.

115 - conceptuellement :

(34) a. marxisme: $<\mathrm{x}$ : ce que $><\mathrm{y}:$ Marx $><$ valorise $>$

b. marxisme : <valorisation $>$ de

$<$ b1 : le capitalisme est une aliénation ;

$\mathrm{b} 2:$ la révolution prolétaire ; etc.>'

116 La variation conceptuelle montre que l'ISME peut être défini comme la valorisation non pas d'une seule idée mais d'un réseau d'idées liées entre elles par diverses relations (causes, conséquences, conditions, buts, moyens, antériorité, partie-tout, concessions ...). Les variations conceptuelle et rhétorique ne sont pas toujours faciles à distinguer.

117 Nous en avons terminé avec cette première section. Pour la section 2, nous fonctionnerons avec la variable $<b>$ car il ne sera plus question de morphologie. Si l'on veut, en revanche, continuer l'analyse morphologique, il faudra maintenir les variables $<\mathrm{x}\rangle$ et $\langle\mathrm{y}>$ (pour voir si leur prétention au statut de base est valable ou non). La description sémantique des ISMEs à sens valorisationnel va maintenant se poursuivre en pénétrant dans une autre discipline, à savoir la pragmatique. 


\section{Description du sens valorisationnel en contexte de rivalité}

Dans la section précédente, nous avons proposé une description sémantique assez formalisée du sens valorisationnel des ISMEs. Des cas particuliers peuvent, çà et là, soulever des questions mais le schéma général a été posé. Dans cette section, nous allons poursuivre cette description sémantique en y intégrant un autre fait: l'interprétation valorisationnelle d'un ISME est toujours opérée en contexte de rivalité. Dans un premier temps nous développerons ce que nous appelons contexte de rivalité puis nous proposerons une manière d'intégrer ce contexte dans la description sémantique des ISMES.

\subsection{Le contexte de rivalité : de l'extralinguistique au linguistique}

119 Livrons-nous d'abord à un exercice d'imagination. Imaginons-nous dans le rôle d'un Parisien qui décide de passer une partie de ses vacances dans un village français. Un jour que nous profitons du charme des lieux, notre attention est attirée par les discussions animées des locaux sur la place du village. Ils ont l'air de se demander comment faire prospérer l'économie du village qui est fort dégradée. Différentes idées sont proposées dont deux qui semblent retenir particulièrement l'attention de la population. La première serait d'exploiter le terroir du lieu pour se spécialiser dans la culture et le commerce de la pomme. L'autre consisterait à faire la même chose mais avec la poire. Au cours de leurs discussions, les villageois en arrivent à donner des noms aux différents acteurs impliqués. Ils appellent le partisan de la première solution 'pommiste et sa solution ${ }^{\circ}$ pommisme et le partisan de la seconde solution ${ }^{\circ}$ poiriste et sa solution ${ }^{\circ}$ poirisme. Ce qui nous semble pertinent à examiner linguistiquement est la question suivante : l'interprétation valorisationnelle de ${ }^{\circ}$ pommisme et ${ }^{\circ}$ poirisme auraitelle pu être opérée si le contexte de rivalité que nous avons décrit (ou un autre, peu importe) n'avait pas existé ? La réponse est toujours non pour les ISMEs à valorisation modale et presque toujours non pour les ISMEs à valorisation attitudinale ${ }^{23}$. Nous avons vérifié cette réponse sur corpus dans Agabalian (2019b) : sauf rares et contestables exceptions (note 22), il est impossible qu'un ISME puisse être interprété au sens d'une valorisation $d e<b>$ en dehors d'un contexte de rivalité entre l'entité que représente $<b>$ (une personne, une idée, une valeur...) et une ou plusieurs autre(s) entité(s) (une autre personne, une autre idée, une autre valeur...). On peut donc dire qu'il y a une condition pragmatique à l'actualisation de l'interprétation valorisationnelle d'un ISME. Dans la mesure où cette hypothèse est déjà vérifiée, ce n'est pas la question de sa vérification qui sera posée ici mais celle de son origine : comment se fait-il que l'interprétation valorisationnelle des ISMEs s'opère (quasi-)systématiquement en contexte de rivalité ?

1) L'origine extralinguistique de l'interprétation valorisationnelle en contexte de rivalité :

Pour reprendre Roché (2011), la suffixation en -isme permet de construire des noms qui répondent à trois quêtes humaines : la quête de ce qui est vrai (par ex. nominalisme), de ce qui est bien (par ex. féminisme) et de ce qui est beau (par ex. impressionnisme). Or, il n'a échappé à personne que les hommes ne sont pas souvent d'accord à propos de ce qui est vrai, bien ou beau. Il n'y a donc rien d'étonnant à ce que la description 
sémantique d'un ISME valorisationnel nécessite que l'on situe son sens dans un contexte de rivalité.

Beaucoup d'ISMEs dénommant des doctrines, des religions, des mouvements divers et variés sont, de fait, apparus dans des contextes historiques de débats, de disputes, de dissidences, voire de violence. C'est un fait qui a été souvent rappelé et/ou illustré par des historiens ou des sociologues. Ainsi, dans le domaine de la religion Éliade aborde les désaccords qui sont à l'origine de bon nombre d'ISMEs dans le christianisme (Éliade \& Couliano, 2016:99-128). Dans le domaine de l'art, on pourra se référer à l'ouvrage de Boschetti consacré aux ISMES eux-mêmes, particulièrement au passage où elle décrit comment réalisme a été formé explicitement par hostilité au romantisme (Boschetti, 2014 : 40-42). Hopfl $(1983: 4-8,12)$ considère, de son côté, qu'il y a eu, dans l'histoire, deux périodes de pic de production de nouveaux ISMEs: le $16^{\mathrm{e}}-17^{\mathrm{e}}$ siècle avec la prolifération de doctrines chrétiennes dissidentes (des " hérésies ») et le $19^{\mathrm{e}}$ siècle où les ISMES sont devenus les armes rhétoriques par excellence de la nouvelle figure de l'« intellectuel ». Kurunmäki \& Marjanen (2018 : 267-269), qui ont récemment dirigé un numéro de Journal of political ideologies consacré aux ISMEs, ajoutent qu'une autre période critique pour les ISMES est le début du $20^{\mathrm{e}}$ siècle lorsqu'ils sont assimilés aux idéologies totalitaires en Europe.

\section{2) L'intégration au système linguistique :}

Ce contexte de rivalité n'est pas resté extérieur au système de la langue. Notre hypothèse est la suivante: l'interprétation en contexte de rivalité s'est attachée formellement (et très tôt selon nous) à la suffixation en -isme de sorte que la perception auditive (ou visuelle) de la forme en -isme a tendance à déclencher, dans l'esprit du locuteur, l'imagination d'un contexte de rivalité. Même le locuteur ignorant les conditions sociohistoriques de l'actualisation d'un ISME en viendrait à reconstituer un contexte de rivalité imaginaire à peu près plausible (comme nous l'avons fait avec opommisme et ${ }^{\circ}$ poirisme) pour satisfaire les conditions de bonne actualisation et de bonne interprétation de cet ISME. C'est une hypothèse psycholinguistique qui pourrait être vérifiée/réfutée en soumettant aux locuteurs des ISMEs néologiques (dans lesquels ils reconnaissent facilement les bases) et en leur demandant de commenter le sens de ces ISMES ou d'imaginer des contextes où on pourrait les employer (comme nous l'avons fait sur nous-mêmes avec ${ }^{\circ}$ pommisme et ${ }^{\circ}$ poirisme dans une démarche introspective).

Si cette hypothèse psycholinguistique se vérifie, elle posera des questions éthiques quant à l'acte d'ismiser quelque chose. À chaque fois que nous commettons l'acte linguistique d'ismiser une pratique, une tendance, une idée (par exemple si nous ismisons la consommation de la viande en parlant de viandisme), nous prenons la responsabilité de déclencher, dans l'esprit de notre interlocuteur, l'imagination d'une rivalité, voire d'un conflit (par exemple entre le fait de se nourrir avec de la viande et le fait de se nourrir sans, entre ceux qui en consomment et ceux qui n'en consomment pas). Nous disons bien "déclencher " car il est tout à fait possible qu'avant de nous avoir parlé, notre interlocuteur n'avait pas nécessairement envisagé la situation sous l'angle de la rivalité ou du conflit et notre ismisation des choses a implanté ce point de vue dans son esprit.

Du reste, l'ismisation néologique de quelque chose ou même l'utilisation d'un ISME installé, peut parfois créer une situation qui rappelle, par certains aspects seulement, ce que certains logiciens ont appelé l'opacité référentielle (Apothéloz, 2010). L'exemple 
souvent donné dans la littérature est l'énoncé CEdipe voulait épouser sa mère. Il se trouve que cet énoncé n'est vrai que pour un énonciateur qui sait que les expressions la femme qu'ÆEdipe voulait épouser et la mère d'ÆEdipe sont co-référentielles. Mais cette coréférentialité, đEdipe l'ignore, ce qui place l'énonciateur en situation de supériorité par rapport à lui. Quelque chose de proche peut se produire lorsqu'un locuteur, par exemple hostile à la consommation de la viande, emploie les noms viandisme et viandiste. Ce locuteur peut raisonner comme si le consommateur de viande et le viandiste étaient deux expressions co-référentielles et qu'il le savait, alors que ledit consommateur de viande, de son côté, ne serait pas au courant. La différence avec l'exemple d'œdipe est que cette co-référentialité n'est pas factuelle. Elle repose sur une indifférenciation inférée entre un agent non sujet modal (le consommateur) et un agent sujet modal (l'avocat de la consommation). Cette indifférenciation n'est pas systématique mais elle est suffisamment tentante pour que certains locuteurs opposés à la consommation de la viande ressentent le besoin de préciser qu'il faut réserver les termes viandisme et viandiste au fait seulement d'être l'avocat de la consommation de la viande ${ }^{24}$.

Nous soutenons donc que, même si la rivalité est d'origine extralinguistique, sa conception a fini par devenir une contrainte formelle de l'interprétation valorisationnelle des ISMEs. Mais il reste encore à définir qui sont les rivaux. Et c'est peut-être là que notre conception du contexte de rivalité rompt le plus avec ses origines extralinguistiques. Chez nous, ce contexte n'est pas dit «de rivalité » parce qu'il consiste en une opposition entre les partisans d'une entité et les opposants à cette entité ${ }^{25}$. Il est dit « de rivalité " parce qu'il consiste en une opposition entre l'entité que représente $<\mathrm{b}>$ et une ou plusieurs autre(s) entité(s). Pour évolutionnisme par exemple, le contexte de rivalité n'est pas le contexte où s'opposent les évolutionnistes et les fixistes. Il est celui où s'opposent, par exemple, l'idée exprimée par $<\mathrm{b}:$ les espèces sont le résultat d'une évolution $>$ et l'idée exprimée par $<\mathrm{d}$ : les espèces sont apparues telles quelles de novo>. Pour féminisme, il en va de même : il n'est pas celui où s'opposent les féministes et les anti-féministes, mais celui où s'opposent, par exemple, l'idée exprimée par $<b$ : la femme est l'égale de l'homme> et l'idée exprimée par $<d$ : la femme est inférieure à l'homme>. Cela signifie donc que, pour nous, la relation de rivalité ne concernera pas les opposants et les partisans humains mais les expressions linguistiques (propositions ou syntagmes) qui instancient $<b>$ et son rival, qu'on notera $<\mathrm{d}>$. En étudiant l'opposition des matériels linguistiques qui instancient $<\mathrm{b}>$ et $<\mathrm{d}>$, nous restons dans le domaine de la linguistique alors que l'opposition entre partisans et opposants nous conduirait vers la sociologie.

Ayant précisé notre conception du contexte de rivalité, il reste maintenant à expliquer comment nous comptons intégrer ce contexte dans une description sémantique formalisée des ISMES. Cette entreprise comprendra deux étapes: d'abord nous définirons la nature de l'opposition entre $<\mathrm{b}>$ et $<\mathrm{d}>(2.2)$, puis nous définirons la nature des contextes de rivalité dans lesquels ils sont situés (2.3).

\subsection{La nature de l'opposition entre $<b>$ et $<d>$}

Nous distinguerons deux natures d'opposition :

1) une opposition sémantico-logique : ce sont les relations d'opposition classiques d'antonymie, de complémentarité et d'inversion ; 
131 2) une opposition pragmatique : c'est une relation d'opposition qui peut s'établir entre deux expressions linguistiques lorsque ces expressions désignent des entités (des personnes, des idées, des institutions, des disciplines scientifiques, des objets matériels, des nations, etc.) qui sont en rivalité ou qui ont été mises en rivalité dans un contexte donné et dont on constate ou s'imagine qu'elles cherchent à s'imposer l'une au détriment de l'autre.

Le contexte de rivalité est toujours au minimum une opposition pragmatique entre ce que représentent $<b>$ et $<d>$, qu'il s'agisse d'humains $(<b:$ les femmes $>$ vs $<d$ : les hommes $>$ pour féminisme; $<\mathrm{b}:$ le peuple $>\mathrm{vs}<\mathrm{d}$ : l'élite ; les étrangers $>$ pour populisme ; $<\mathrm{b}:$ moi $>$ vs $<\mathrm{d}$ : autrui $>$ pour égoïsme), d'idées ( $<\mathrm{b}:$ la femme est l'égale de l'homme> vs $<\mathrm{d}$ : la femme est inférieure à l'homme> pour féminisme; $<\mathrm{b}$ : il n'y a qu'un seul dieu $>$ vs $<\mathrm{d}$ : il y a plusieurs dieux $>$ pour monothéisme) ou autre $(<\mathrm{b}:$ le style $>\mathrm{vs}<\mathrm{d}$ : le fond $>$ pour stylisme). Cette opposition pragmatique peut se cumuler parfois avec une opposition sémantico-logique, toujours entre $<\mathrm{b}>$ et $<\mathrm{d}>$.

\subsubsection{Opposition uniquement pragmatique}

Il y a longtemps déjà Martin (1976: 70), Pohl (1970:188) ou Duchaček (1965: 64) notaient qu'une "antonymie » d'origine extralinguistique peut finir par s'instaurer entre deux unités qui pourtant, du point de vue de leur sens premier, ne sont pas des antonymes: corbeau et renard, Racine et Corneille, ville et campagne, collaboration et résistance, etc. Ce sont leurs exemples. On pourrait ajouter thé et café, cinéma et théâtre, PSG et OM, science et art, chien et chat, etc. Généralement, il s'agit d'unités incompatibles au sens de Lyons (1978 : 233 sq.) ou de Cruse (1986 : 93 sq.), c'est-à-dire - pour résumer sauvagement - de deux co-hyponymes qui, par définition, s'excluent paradigmatiquement (si X est du thé, X ne saurait être du café). Notre exemple avec l'opposition entre pomme et poire relève très précisément de ce phénomène: deux lexèmes qui, à l'origine, sont seulement des incompatibles finissent par s'antonymiser dans un contexte donné et dans le langage d'un groupe de locuteurs donné. Sur un plan théorique, l'usage du terme "antonymie " pose problème pour caractériser cette opposition mais nous ne pouvons pas développer cette réflexion ici (voir Lyons, 1970 : 353 ; Cruse, 1986 : 204 ; Picoche, 1992 : 102). Disons seulement que nous continuerons simplement à parler d'opposition pragmatique.

Cette opposition pragmatique est tout ce dont a besoin la suffixation en -isme pour construire un sens valorisationnel. Parmi les ISMEs dont l'interprétation valorisationnelle peut être opérée dans le cadre d'une opposition pragmatique seule, un exemple très représentatif nous semble être capitalisme. Comme le montre clairement Rainer (2018: 54 sq.), le lexème capitalisme a d'abord servi à désigner des activités de rente et d'investissement et son sens '<valorisation $><x$ : du capital' n'est apparu que lorsque le travail et le capital ont été mis en rivalité. Cette rivalité s'est déposée dans le sens des lexèmes capital et travail jusqu'à en faire des "antonymes" pour Rainer (des opposés pragmatiques pour nous). Mais on peut citer beaucoup d'autres exemples d'ISMEs dont l'interprétation valorisationnelle peut être opérée dans le cadre d'une opposition pragmatique seule. Le sens de tous les ISMEs construits à partir d'un nom de discipline (biologisme, économisme, historicisme, psychologisme, sociologisme...) repose entièrement sur la mise en rivalité des disciplines scientifiques pour déterminer laquelle est la plus fondamentale : 
(32) Biologisme: Doctrine qui présente les phénomènes psychologiques et sociologiques comme ayant toujours une assise biologique. (TLFi)

(33) Psychologisme: Tendance à faire prévaloir le point de vue de la psychologie sur celui d'une autre science, dans un domaine commun (opposé à sociologisme, etc.). (PR17)

\subsubsection{Opposition pragmatique doublée par une opposition sémantico-logique}

Cette opposition pragmatique peut, parfois, se cumuler avec une opposition sémanticologique entre $<b>$ et $\langle d>$. Même si celle qui est la plus importante et la seule nécessaire reste l'opposition pragmatique.

1) opposition pragmatique + antonymie ou inversion ${ }^{26}$

Prenons les exemples de pacifisme et de populisme. Comme instanciations possibles pour leur $<\mathrm{b}>$ et $<\mathrm{d}>$ respectifs, on trouve les syntagmes nominaux suivants :

pacifisme $<\mathrm{b}$ : la paix $>$; $<\mathrm{d}$ : la guerre $>$

populisme $<\mathrm{b}$ : le peuple $>$; $<$ : l'élite $>$

Comme le veut notre conception du contexte de rivalité, il y a une opposition pragmatique entre $<b>$ et $<d>$ en ce sens qu'ils représentent des entités mises en rivalité et dont on constate ou s'imagine qu'elles cherchent à s'imposer l'une au détriment de l'autre (même avec un $<b>$ comme la paix). Mais, en plus de cette opposition pragmatique, il y a aussi une opposition sémantico-logique entre $\langle\mathrm{b}\rangle$ et $\langle\mathrm{d}\rangle$ : entre la paix et la guerre on peut reconnaître une relation d'antonymie et entre le peuple ${ }^{27}$ et l'élite une relation d'inversion. Précisons que dans certaines de ces oppositions (comme peuple et élite), il est difficile de voir une dimension sémantico-logique qui serait vraiment distincte d'une dimension pragmatique.

2) opposition pragmatique + complémentarité 28

Imaginons, par exemple, pour évolutionnisme les instanciations suivantes $:<\mathrm{b}:$ les espèces sont le résultat d'une évolution> et $<d$ : les espèces sont apparues telles quelles de novo $>$. $<b>$ et $<d>$ sont forcément des opposés pragmatiques mais $<b>$ est également complémentaire par implication avec $\langle\mathrm{d}>$, soit $b \supset \sim d$ qu'on peut schématiser de la manière suivante :

Les espèces...

$<\mathrm{b}$ : sont le résultat d'une évolution $>\quad<\mathrm{d}$ : sont apparues telles quelles de

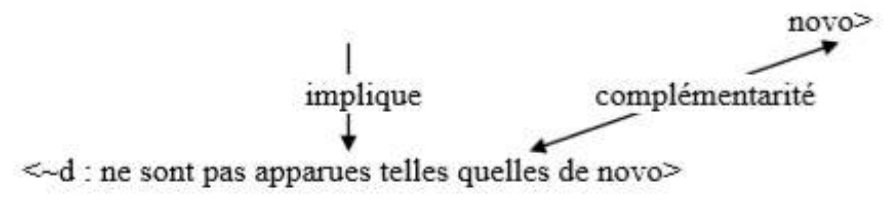

L'explicitation de ces relations d'opposition entre l'objet valorisé $(<b>)$ et son rival $(<\mathrm{d}>)$ n'est pas une option dans la description sémantique des ISMES valorisationnels. Elle constitue, bien au contraire, une étape impérative de l'analyse. L'identification d'une opposition pragmatique ainsi que la mise à contribution des relations d'opposition lexicale classiques doivent nous permettre de satisfaire cette étape pour déboucher sur la suivante qui est la reconstitution des contextes de rivalité dans lesquels le sens valorisationnel des ISMEs est nécessairement situé. 


\subsection{Les contextes de rivalité reconstitués}

Une fois l'opposition entre $<\mathrm{b}>$ et $<\mathrm{d}>$ reconnue et explicitée, il faut situer $<\mathrm{b}>$ et $<\mathrm{d}>$. En l'occurrence, il faut les situer dans des contextes de rivalité qui, dans notre approche, ne sont pas nécessairement des situations historiquement constatées mais qui sont des reconstitutions permettant de satisfaire les conditions qui rendent pertinente l'attribution d'un sens valorisationnel à un ISME.

\subsubsection{Le désaccord}

Le contexte de désaccord est le premier des trois contextes de rivalité. Il est exclusivement dédié à la description de la valorisation modale de type épistémique et assertif (voir schéma 1). Il permet de décrire cette valorisation en la situant dans un contexte où s'opposent l'énonciation de la certitude que $<b>$ est ce qui est vrai et l'énonciation de la certitude que c'est $<\mathrm{d}>$ qui est vrai. Autrement dit, le désaccord est un contexte où s'opposent des actes assertifs (au sens de Searle (1982)).

Ses caractéristiques sont les suivantes (les quatre premières doivent se cumuler) :

1) Dans une périphrase dénominative (Kleiber, 1984), la <valorisation> doit être instanciée par un verbe locutoire qui, par son sens lexical, est un acte assertif ainsi qu'une modalisation épistémique positive de $<\mathrm{b}>$. Typiquement, affirmer que :

(34) On appelle nominalisme quelque chose qui affirme que $<b>29$

2) Toujours dans une périphrase dénominative, $<b>$ (et $<d>$ aussi) doit être instancié par un contenu propositionnel sous la forme d'une proposition ou d'un syntagme :

(35) On appelle monothéisme quelque chose qui affirme qu'il n'existe qu'un dieu

(ou) l'existence d'un seul dieu

3) $<b>$ et $<d>$ sont à la fois des opposés pragmatiques et des complémentaires. <b> est complémentaire avec $<\mathrm{d}>$ par implication, une situation qu'on retrouve souvent dans les actes de réfutation (Moeschler, 1982 : 71). Par exemple pour immanentisme:

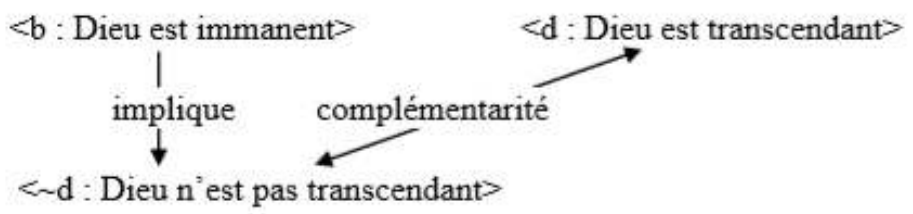

$148 \mathrm{Si}<\mathrm{b}>$ est négatif (surtout à cause d'un préfixe négatif dans le radical), sa complémentarité avec $<\mathrm{d}>$ se fait directement. C'est le cas pour athéisme :

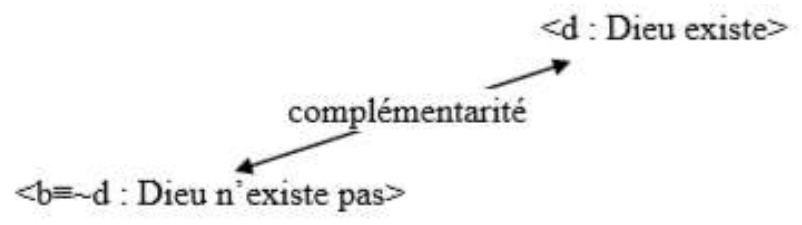

149 4) La valorisation de <b> est obligatoirement une séquence dans un dialogue reconstitué comprenant trois actes de langage: une assertion initiale, une réfutation de cette assertion initiale et une rectification de cette assertion initiale.

150 a) $\mathrm{Si}<\mathrm{b}>$ n'est pas négatif, sa valorisation correspond à l'acte de rectification. Par exemple, pour immanentisme: 


\begin{tabular}{|c|l|l|}
\hline $\mathrm{N}^{\circ}$ & Désaccord & Exemples pour immanentisme \\
\hline 1 & $\begin{array}{l}\text { assertion initiale } \mathrm{de}<\mathrm{d}> \\
2\end{array}$ & $\begin{array}{l}\text { réfutation } \mathrm{L}-\mathrm{E}_{1} / \mathrm{E}_{1}{ }^{30} \text { affirme que Dieu est transcendant } \\
(\text { assertion } \mathrm{de}<\sim \mathrm{d}>\text { ) }\end{array}$ \\
\hline 3 & $\begin{array}{l}\text { rectification } \mathrm{par}<\mathrm{b}> \\
(\text { assertion } \mathrm{de}<\mathrm{b}>\text { ) }\end{array}$ & $\mathrm{L}-\mathrm{E}_{2} / \mathrm{E}_{2}$ affirme que Dieu n'est pas transcendant \\
\hline
\end{tabular}

Le niveau (3) correspond au sens valorisationnel d'immanentisme tel qu'on le trouve représenté, par exemple, dans les définitions de dictionnaire. Mais ce sens valorisationnel est le résultat d'une interprétation en contexte de rivalité puisqu'il est, littéralement, une rectification qui répond à une assertion initiale réfutée ${ }^{31}$. Ce qui montre aussi que le sens d'immanentisme, comme celui de tous les ISMEs interprétés en contexte de désaccord, est polyphonique.

b) $\mathrm{Si}$ <b> est négatif (surtout à cause d'un préfixe négatif dans le radical), sa valorisation correspond à la réfutation de l'assertion initiale :

\begin{tabular}{|c|l|l|}
\hline $\mathrm{N}^{\circ}$ & Désaccord & Exemples pour athéisme \\
\hline 1 & $\begin{array}{l}\text { assertion initiale } \mathrm{de}<\mathrm{d}> \\
2\end{array}$ & $\begin{array}{l}\text { Léfutation } \mathrm{de}<\mathrm{d}> \\
(\text { assertion } \mathrm{de}<\sim \mathrm{d}>\text { ) }\end{array}$ \\
\hline 3 & $\begin{array}{l}\text { rectification } \mathrm{par}<\mathrm{b}> \\
(\text { assertion } \mathrm{de}<\mathrm{b}>\text { ) }\end{array}$ & $\varnothing$ \\
\hline
\end{tabular}

Il est tout à fait logique que la rectification ne soit pas nécessaire. Comme $<b>$ est équivalent à $<\sim \mathrm{d}>$, l'assertion de $c e<\sim \mathrm{d}>$ (donc la réfutation) équivaut à l'assertion de $<b>$. Bien sûr, dans le cas d'athéisme, on pourrait imaginer que la rectification ne soit pas vide mais instanciée par un deuxième $<b>$, par exemple $<b$ : la matière suffit à rendre compte du monde $>$ ( $8^{\mathrm{e}}$ type de $<\mathrm{b}>$ dans le tableau des principaux liens entre $<\mathrm{b}>$ et $\langle x>$ ). Cependant, ce qui nous intéresse ici est de déterminer le premier acte du contexte dialogal où athéisme est donné comme signifiant la valorisation de quelque chose. Et ce premier acte reste la réfutation.

5) Les ISMEs dont l'interprétation valorisationnelle est opérée en contexte de désaccord peuvent s'organiser en paradigmes sémantiques dynamiques au sein desquels on 
progresse suivant un enchâssement de réfutations et de rectifications à partir d'une question de départ :

a) Une logique en « Non »: C'est A / Non ce n'est pas A, c'est B.

Image

10018C300000842500004A4F6754B412D91EFDFD.emf

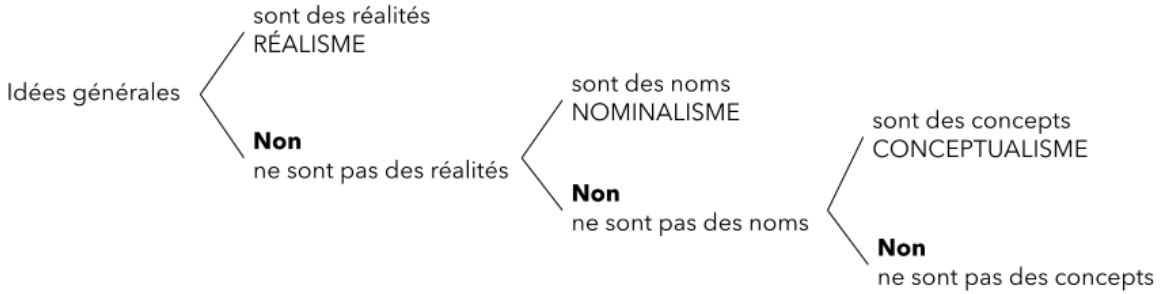

Schéma 2. Exemple de paradigme de désaccord en « Non » 32

b) Une logique en « Si » : Ce n'est pas A / Si c'est A mais ce n'est pas B.

Image

1001BD040000842500004A4F971077C999E96A9F.emf

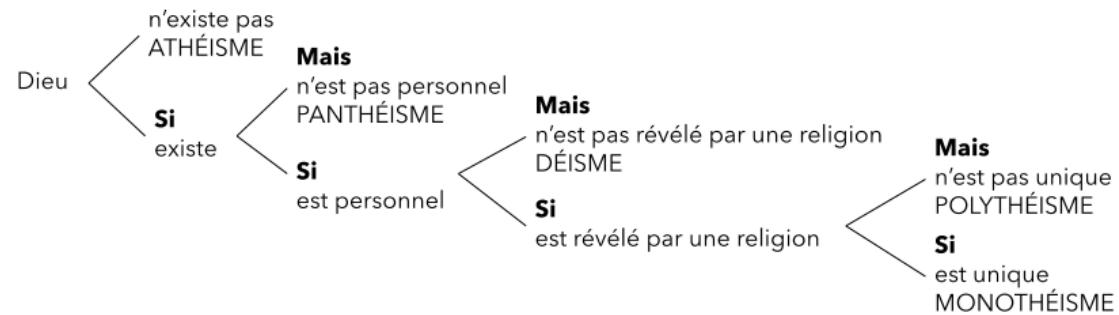

Schéma 3. Exemple de paradigme de désaccord en « Si »

\subsubsection{La rupture}

157 Le contexte de rupture est exclusivement dédié à la description de la valorisation modale de type boulique/déontique et directif (voir schéma 1). Il permet de décrire cette valorisation en la situant dans un contexte où s'opposent l'énonciation de la volonté que $<\mathrm{b}>$ advienne et l'énonciation de la volonté que $<\mathrm{d}>$ advienne. Autrement 
dit, on passe d'un contexte de rivalité opposant des certitudes (désaccord) à un contexte opposant des volontés (rupture).

\begin{tabular}{|c|l|l|}
\hline $\mathrm{N}^{\circ}$ & Rupture & Exemples pour autonomisme \\
\hline 1 & $\begin{array}{l}\text { directif initial visant à faire } \\
\text { advenir }<\mathrm{d}>\end{array}$ & $\begin{array}{l}\mathrm{L}-\mathrm{E}_{1} / \mathrm{E}_{1} \text { exige qu'une région donnée soit } \\
\text { dépendante de l'État }\end{array}$ \\
\hline 2 & $\begin{array}{l}\text { refus de }<\mathrm{d}> \\
3\end{array}$ & $\begin{array}{l}\mathrm{L}-\mathrm{E}_{2} / \mathrm{E}_{2} \text { refuse que cette région soit dépendante } \\
\text { de l'État }\end{array}$ \\
\hline $\begin{array}{l}\text { directif alternatif visant à faire }<\mathrm{b}> \\
\mathrm{L}-\mathrm{E}_{2} / \mathrm{E}_{2} \text { réclame que cette région soit autonome }\end{array}$ & \multicolumn{2}{|c}{} \\
\hline
\end{tabular}

4) La valorisation de $<b>$ est obligatoirement une séquence dans un dialogue reconstitué comprenant trois actes de langage : une acte directif initial, un refus adressé à cet acte directif et un acte directif alternatif.

a) $\mathrm{Si}<\mathrm{b}>$ n'est pas négatif, sa valorisation correspond à l'acte directif alternatif :

(37) On appelle libéralisme quelque chose qui réclame qu'on étende les libertés individuelles

3) $<b>$ et $<\mathrm{d}>$ sont à la fois des opposés pragmatiques et des complémentaires dans les mêmes conditions que pour le contexte de désaccord:

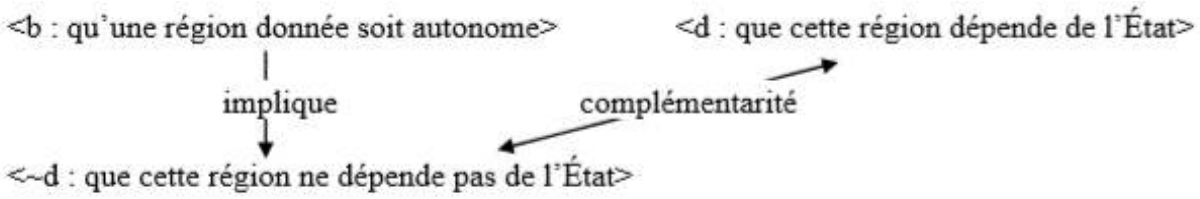

b) $\mathrm{Si}<\mathrm{b}>$ est négatif (surtout à cause d'un préfixe négatif dans le radical), exemple avec anarchisme:

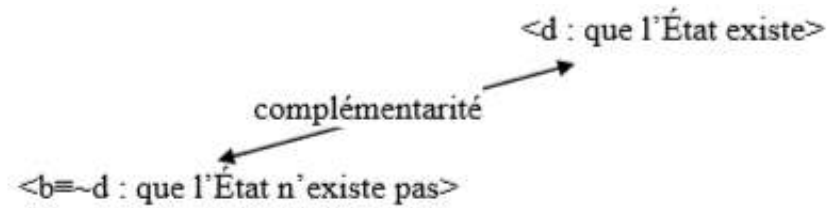


Comme pour le sens des ISMEs interprétés en contexte de désaccord, celui des ISMES interprétés en contexte de rupture est polyphonique.

b) $\mathrm{Si}$ <b> est négatif (surtout à cause d'un préfixe négatif dans le radical), sa valorisation correspond au refus. Exemple avec anarchisme :

\begin{tabular}{|c|l|l|}
\hline $\mathrm{N}^{\circ}$ & Rupture & Exemples pour anarchisme \\
\hline 1 & directif initial visant à faire advenir $<\mathrm{d}>$ & $\mathrm{L}-\mathrm{E}_{1} / \mathrm{E}_{1}$ exige que l'État existe \\
\hline 2 & refus de $<\mathrm{d}>$ & $\mathrm{L}-\mathrm{E}_{2} / \mathrm{E}_{2}$ refuse que l'État existe \\
\hline 3 & directif alternatif visant à faire advenir $<\mathrm{b}>$ & $\varnothing$ \\
\hline
\end{tabular}

On peut imaginer que l'acte directif alternatif soit rempli par un deuxième $<b>$, par exemple $<b$ : que l'individu soit le seul souverain $>\left(8^{e}\right.$ type de $<b>$ ). Nous ferons la même remarque que pour athéisme: il n'y a rien d'incorrect à procéder ainsi mais ce qui nous intéresse ici est de déterminer le premier acte du contexte dialogal où anarchisme est donné comme signifiant la valorisation de quelque chose et ce premier acte reste le refus.

5) Les ISMES dont l'interprétation valorisationnelle est opérée en contexte de rupture peuvent aussi s'organiser en paradigmes sémantiques mais, contrairement au contexte de désaccord, ces paradigmes ne sont pas dynamiques mais statiques. En d'autres termes, il n'y a pas de progression de gauche à droite par enchâssement de refus et d'actes directifs alternatifs. À la place, on trouve une division statique en deux parties : d'un côté, l'acte directif initial et, de l'autre, les actes directifs alternatifs consécutifs au refus mais non pas enchâssés les uns dans les autres - cela produirait des lectures aberrantes - plutôt alignés sur le même plan : 


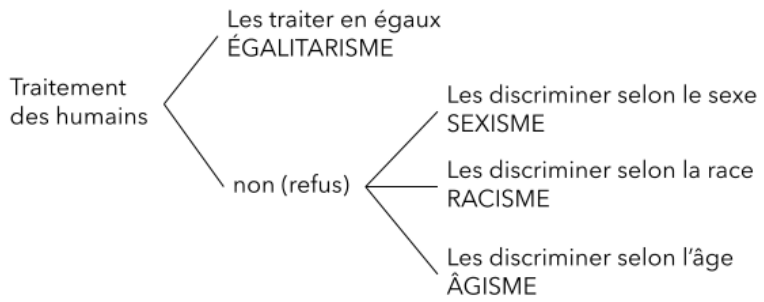

Schéma 4. Exemple de paradigme de rupture ${ }^{33}$

\subsubsection{La hiérarchie}

Le contexte de hiérarchie est ambivalent: il permet de décrire la valorisation attitudinale quand il intervient seul et la valorisation modale quand il est combiné avec le désaccord ou la rupture. Contrairement à ces derniers, il n'est pas un contexte dialogal où s'opposent des actes d'énonciation mais une comparaison où s'opposent des hiérarchisations inverses.

Ses caractéristiques sont les suivantes (les quatre premières doivent se cumuler):

1) Dans une périphrase dénominative, la <valorisation> doit être instanciée par un verbe qui n'est pas locutoire par son sens lexical et qui exprime une supériorité $d e \quad b \mathrm{~b}>$ :

(39) On appelle psychologisme quelque chose qui fait prévaloir $<b>$

2) Toujours dans une périphrase dénominative, $\langle b>$ (et $<d>$ aussi) ne doit pas être instancié par un contenu propositionnel :

(40) On appelle psychologisme quelque chose qui fait prévaloir la psychologie

3) Toutes les relations d'oppositions entre $<b>$ et $<d>$ sont possibles ${ }^{34}$ :

a) Seulement des opposés pragmatiques : psychologisme

\section{$+<\mathrm{b}:$ la psychologie $>$
$\downarrow-<\mathrm{d}:$ la sociologie $>$}

176 b) Des opposés pragmatiques et des complémentaires : sexisme

$$
\begin{aligned}
& +<\mathrm{b}: \text { les hommes }> \\
& \downarrow-<\mathrm{d}: \text { les femmes }>
\end{aligned}
$$

177 c) Des opposés pragmatiques et des antonymes : pacifisme 


\section{$+<$ b : la paix $>$ \\ $-<\mathrm{d}:$ la guerre $>$}

d) Des opposés pragmatiques et des inverses : populisme

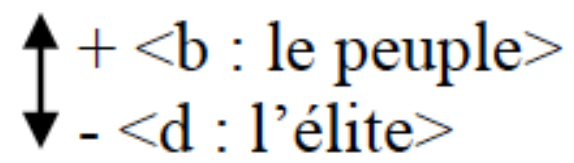

4) La $<$ valorisation $>$ de $<b>$ est une supériorisation de $\langle b>$. Cette supériorisation se fait au sein d'un contexte qui comprend l'infériorisation $\mathrm{de}<\mathrm{d}>\mathrm{et}$, surtout, qui comprend la mise en place d'une échelle de valeur :

\begin{tabular}{|c|c|c|}
\hline Numéro & Hiérarchie & Exemples pour rationalisme \\
\hline 1 & échelle hiérarchique & échelle de valeur des sources de la connaissance \\
\hline 2 & supériorisation $\mathrm{de}<\mathrm{b}>$ & faire prévaloir la raison \\
\hline 3 & infériorisation de $<\mathrm{d}>$ & minorer l'expérience \\
\hline $\mathbf{N}^{\circ}$ & Hiérarchie & Exemples pour psychologisme \\
\hline 1 & échelle hiérarchique & échelle de valeur des sciences \\
\hline 2 & supériorisation $\mathrm{de}<\mathrm{b}>$ & faire prévaloir la psychologie \\
\hline 3 & infériorisation de $<\mathrm{d}>$ & minorer la sociologie \\
\hline $\mathbf{N}^{\circ}$ & Hiérarchie & Exemples pour spécisme \\
\hline 1 & échelle hiérarchique & échelle de valeur des espèces vivantes \\
\hline 2 & supériorisation $\mathrm{de}<\mathrm{b}>$ & faire prévaloir l'espèce humaine \\
\hline 3 & infériorisation de $<\mathrm{d}>$ & minorer les espèces animales \\
\hline
\end{tabular}


5) Dans une périphrase dénominative, la hiérarchie ([hiér]) peut se combiner avec le désaccord ([dés]) ou la rupture ([rup]), auquel cas la valorisation obtenue est complexe (car deux contextes de rivalité sont enchâssés) et toujours de type modal :

(40) On appelle rationalisme quelque chose qui

affirme[dés] que la raison prévaut sur[hiér] l'expérience

(41) On appelle rationalisme quelque chose qui

préconise[rup] de faire prévaloir[hiér] la raison sur l'expérience socialisme, etc. ; En revanche, quand la hiérarchie intervient seule dans l'interprétation valorisationnelle, le type de valorisation obtenu ne peut être qu'attitudinal :

(42) On appelle carriérisme quelque chose qui consiste à faire prévaloir sa carrière (au détriment de $<\mathrm{d}$ : l'éthique professionnelle>)

(43) On appelle égoïsme quelque chose qui consiste à faire prévaloir sa propre personne (au détriment de $<\mathrm{d}$ : autrui $>$ )

Le contexte de hiérarchie ne permet toutefois pas de décrire toutes les valorisations attitudinales. Les croyances ou les volontés non-énoncées ainsi que les affects (voir schéma 1) peuvent, parfois, ne pas se laisser décrire par le contexte de hiérarchie et nécessiter l'élaboration d'autres contextes de rivalité. L'objectif, ici, n'est pas de passer en revue toutes les rivalités possibles et imaginables mais de donner les principales d'après notre corpus.

6) Les ISMEs dont l'interprétation valorisationnelle a été opérée dans un contexte de hiérarchie (avec ou sans combinaison avec le désaccord ou la rupture) peuvent s'organiser en paradigmes sémantiques statiques dont l'unité est assurée par une grande opposition notionnelle :

a) nous vs eux : communautarisme, ethnocentrisme, eurocentrisme, nationalisme, etc. ;

b) le sensible vs l'insensible : matérialisme, immatérialisme, sensualisme, sensationnisme, spiritualisme, etc. ;

c) l'individu vs le groupe: collectivisme, communisme, individualisme, libéralisme,

d) l'individu vs l'État : anarchisme, étatisme, individualisme, libéralisme, etc. ;

e) le passé vs l'avenir ou la conservation vs la transformation : conservatisme, fondamentalisme, intégrisme, modernisme, passéisme, progressisme, traditionalisme, etc.

Pour terminer, nous ferons quatre observations :

1) Les trois contextes de rivalité présentés ici ne constituent pas une typologie de noms suffixés en -isme. Un même ISME peut, en effet, voir son sens valorisationnel construit dans un ou plusieurs de ces contextes, alternativement ou de manière combinée. Ces contextes sont donc une gamme, non-exhaustive, de possibilités que nous avons identifiée dans notre corpus et au sein de laquelle les locuteurs peuvent, jusqu'à un certain point, faire des choix d'interprétation.

2) Deux préfixes jouent un rôle important dans l'interprétation en contexte de rivalité. D'abord anti-. On comprend pourquoi, avec son sens adversatif, il produit naturellement un nom à partir d'un ISME sans avoir besoin d'un adjectif (1.3.3) : comme l'interprétation valorisationnelle d'un ISME se fait en contexte de rivalité, elle crée toujours en creux une position "adverse » à cet ISME, un anti-ISME. L'autre préfixe important est néo-. Il peut signifier 'nouveau' au sens aspectuel: revitalisation d'un ancien ISME (néoclassicisme) ou perduration d'un ISME sous une nouvelle forme 
(néocolonialisme). Mais aussi 'nouveau' au sens argumentatif quand néo-X-isme résulte d'une concession faite à propos d'un ancien ISME (néolibéralisme).

3) Certains ISMES signifient la valorisation d'un $<b>$ qui, d'une manière ou d'une autre, bannit la rivalité ou d'autres notions proches comme la violence ou la domination: éclectisme, égalitarisme, pacifisme, humanisme, œcuménisme, relativisme, etc. Pour autant, l'interprétation valorisationnelle de ces ISMES est bien opérée en contexte de rivalité. Par exemple, pacifisme signifie certes la valorisation d'un $<b>$ qui est tout à fait opposé à la rivalité, en l'occurrence la paix, mais ce sens valorisationnel n'a de raison d'être que dans un contexte où il rivalise avec la valorisation de la guerre ou des solutions militaires. Il convient donc de distinguer la rivalité comme notion (valorisable ou non) et la rivalité comme contexte (qui permet l'interprétation valorisationnelle d'un ISME).

4) Enfin, certains ISMEs dénomment des doctrines ou des mouvements qui se subdivisent en courants internes souvent en désaccord les uns avec les autres. On peut alors se demander comment appliquer ces contextes de rivalité dans un tel cas. Le fait est que souvent ces courants sont distingués les uns des autres dans leur dénomination en ajoutant une épithète à l'ISME. Ainsi féminisme se subdivise en féminisme libéral, féminisme différentialiste, féminisme intersectionnel, féminisme anarchiste, féminisme radical et autres (les appellations sont variables). On peut tout à fait appliquer les contextes de rivalité à ces courants car rien n'interdit de descendre d'un cran dans la taxonomie en opposant non plus $<b>$ et $<d>$ au niveau de l'ISME mais au niveau du courant interne. Si au niveau de l'ISME cela peut mener, parfois, à une opposition avec un autre ISME (par exemple machisme pour féminisme), au niveau du courant interne cela mènera presque toujours à une opposition avec d'autres courants internes (par exemple féminisme différentialiste pour féminisme radical).

\section{Conclusion}

Pour décrire le sens valorisationnel construit par la suffixation en -isme, nous avons commencé par formaliser ce sens au moyen de quatre variables. Deux d'entre elles, $\langle\mathrm{X}\rangle$ et $\langle y>$, doivent être instanciées au moyen d'unités prélevées depuis la forme de l'ISME et rien d'autre que sa forme. Les instanciations des variables <résultat> et <valorisation> sont prélevées depuis un corpus lexicographique, encyclopédique ou un corpus de discours. Nous avons proposé une typologie pour les instanciations de ces deux variables. Face à de nombreux problèmes posés par l'instanciation de $\langle x\rangle$, nous avons décidé de passer par une autre variable, $\langle b>$, dont l'instanciation se fait non plus à partir de la forme mais à partir d'un corpus (comme pour <résultat> et <valorisation>). Nous avons proposé une typologie des variations dans les instanciations de $<b>$ (thématique, rhétorique, conceptuelle). Dans un second temps, nous avons tenté de montrer comment la valorisation de $<b>$ est opérée en le mettant en rivalité avec autre chose. Nous avons distingué deux étapes :

195 1) Expliciter le fait que cette rivalité est toujours une opposition pragmatique mais qu'elle peut aussi se cumuler, parfois, avec une opposition sémantico-logique.

196 2) Reconstituer des contextes d'interprétation qui montrent que la valorisation ne se fait pas sans réfuter (désaccord), refuser (rupture) ou inférioriser (hiérarchie) le rival de $<b>$. 
D'un point de vue plus théorique, on voit que notre approche fait intervenir la morphologie, la sémantique lexicale et la pragmatique. Une telle approche interroge nécessairement sur une économie des disciplines (c'est-à-dire sur une répartition du travail) au sein des sciences du langage pour décrire un seul et même objet d'étude (en l'occurrence ici, un sens).

\section{BIBLIOGRAPHIE}

AGABALIAN Grigory. 2019a. «-Isme : suffixe modal pour la formation de noms de discours ». Travaux de linguistique 79. 43-78.

AGABALIAN Grigory. 2019b. Suffixation en -ISME et construction d'un sens valorisationnel. Thèse de doctorat. Université de Paris.

AMIOT Dany \& DAL Georgette. 2007. « Integrating combining forms into a lexeme-based morphology ». In Booij G. et al., On-line Proceedings of the Fifth Mediterranean Morphology Meeting. Fréjus, septembre 2005. Université de Bologne. 323-336.

ANSCOMBRE Jean-Claude. 1995. « Morphologie et représentation événementielle : le cas des noms de sentiment et d'attitude ». Langue française 105. 40-54.

APOTHÉLOZ Denis. 2010. « L'opacité référentielle : paramètres et statuts discursifs ». In Colas-Blaise M. et al., La question polyphonique ou dialogique en sciences du langage. Recherches linguistiques 31. Université de Metz. 135-155.

BAILLY Anatole. 1935. Dictionnaire grec-français. Paris : Hachette [en ligne : www.tabularium.be/ bailly].

BAESKOW Heike. 2004. Lexical properties of selected non-native morphemes of English. Tübingen : Gunter Narr Verlag.

BAUER Laurie. 1997. « Derivational paradigms ». In G. Booij \& J. Van Maarle, Yearkbook of morphology 1996. Dordrecht : Kluwer. 243-256.

BENIERS JACOBS Elisabeth. 1992. «El sufijo -ismo en el español de México ». In Luna Traill E., Scripta Philologica : in honorem Juan M. Lope Blanch (t.2). Mexico : Universidad Nacional Autónoma de México. 313- 337.

BEVER Thomas G. \& ROSENBAUM Peter S. 1971. « Some lexical structures and their empirical validity ». In Steinberg D. D. \& Jakobovits L. A., Semantics. Cambridge : Cambridge University Press. 586-599.

BONAMI Olivier \& BOYÉ Gilles. 2003. « Supplétion et classes flexionnelles ». Langages 152. 102-126. BONAMI Olivier \& STRNADOVÁ Jana. 2019. «Paradigm structure and predictibility in derivational morphology ». Morphology 29 (2). 167-197.

BOSCHETTI Anna. 2014. ISMES. Du réalisme au postmodernisme. Paris : CNRS Editions.

CHANTRAINE Pierre. 1979 (1933). La formation des noms en grec ancien. Paris : Klincksieck. 
CORBIN Danielle. 1988. « Une hypothèse à propos des suffixes -isme, -ique, -iste du français : la troncation réciproque ». In Landheer R., Aspects de la linguistique française. Hommage à Q.I.M Mok. Amsterdam : Rodopi. 63-74.

CORBIN Danielle. 1999. « Pour une théorie sémantique de la catégorisation affixale ». Faits de langue 14. 65-77.

CRUSE Alan. 1986. Lexical semantics. Cambridge : Cambridge University Press.

CRUSE Alan. 1996. « La signification des noms propres de pays en anglais ». In Rémi-Giraud S. \& Rétat P., Les mots de la nation. Lyon : Presses universitaires de Lyon. 93-102.

DUBoIS Jean. 1962a. Étude sur la dérivation suffixale en français moderne et contemporain. Essai d'interprétation des mouvements observés dans le domaine de la morphologie des mots construits. Paris : Larousse.

DUBOIS Jean. 1962b. Le vocabulaire politique et social en France de 1869 à 1872. Paris : Larousse. DUBOIS Jean \& DUBOIS-CHARLIER Françoise. 1999. La dérivation suffixale en français. Paris : Nathan. DUCHAČEK Otto. 1965. « Sur quelques problèmes de l'antonymie ». Cahiers de lexicologie 6. 55-66. ÉliAde Mircea \& CoUliano Ioan. 2016 (1990). Dictionnaire des religions. Paris : Presses de la Renaissance.

FLAUX Nelly \& StOSIC Dejan. 2015. « Pour une classe des noms d'idéalités ». Langue française 185. 43-57.

FLAUX Nelly \& VAN DE VELDE Danièle. 2000. Les noms en français : esquisse d'un classement. Paris : Ophrys.

HATHOUT Nabil. 2011. « Une approche topologique de la construction des mots : propositions théoriques et application à la préfixation en anti- ». In Roché M. et al., Des unités morphologiques au lexique. Paris : Lavoisier. 251-318.

HATHOUT Nabil \& NAMER Fiammetta. 2014. «Discrepancy between form and meaning in Word Formation : the case of under- and over-marking in French». In F. Rainer et al., Current issues in morphological theory. Morphology and Meaning. Amsterdam: John Benjamins. 177-190.

HEYNA Franziska. 2008. « Sémantisme et potentiel argumentatif des dérivés dénominaux en anti». Discours 2 [en ligne : journals.openedition.org/discours/2022].

HOPFL Harro M. 1983. «Isms ». British journal of political science vol. 3/1. 1-17.

IACOBINI Claudio \& SCALISE Sergio. 2008. « Contraintes de la catégorie de la base et de l'ouput dans la dérivation ». In Fradin B., La raison morphologique. Hommage à la mémoire de Danielle Corbin. Amsterdam/Philadelphie : John Benjamins. 93-112.

KLEIBER Georges. 1984. «Dénomination et relations dénominatives ». Langages 76. 74-94.

KLEIBER Georges \& TAMBA Irène. 1990. «L'hyponymie revisitée : inclusion et hiérarchie ». Langages 98. 7-32.

KURUNMÄKI JUsSi \& MARJANEN Jani. 2018. « Isms, ideologies and setting the agenda for public debate ». Journal of political ideologies vol. 23/3. 256-282.

LIGNON Stéphanie, NAMER Fiammetta \& VILLOING Florence. 2014. « De l'agglutination à la triangulation ou comment expliquer certaines séries morphologiques ». In Neveu F. et al., Actes du $4 e$ Congrès Mondial de Linguistique Française. Paris : Institut de Linguistique Française. 1813-1835. 
LYONS John. 1970 (1968). Linguistique générale, introduction à la linguistique théorique. Paris : Larousse (trad. F. Dubois-Charlier et D. Robinson).

LYONS John. 1978. Éléments de sémantique. Paris : Larousse (trad. J. Durand).

MARCHAND Hans. 1960. The categories and types of present-day English word-formation. Wiesbaden : 0 . Harrassowitz.

MARTIN Robert. 1976. Inférence, antonymie et paraphrase. Paris : Klincksieck.

MEL'CUK Igor, CLAS André \& POLGUÈRE Alain. 1995. Introduction à la lexicologie explicative et combinatoire. Louvain-la-Neuve : Duculot.

MOESCHLER Jacques. 1982. Dire et contredire. Pragmatique de la négation et acte de réfutation dans la conversation. Berne : Peter Lang.

MUÑOZ ARMIJO Laura. 2010. La historia de los derivados en -ismo $e$-ista en el español moderno. Tesis doctoral. Universidad Autónoma de Barcelona.

NAMER Fiammetta. 2013. «Adjectival Bases of French-aliser and -ariser Verbs : Syncretism or Under-specication? ». In Hathout N. et al., Morphology in Toulouse. Selected Proceedings of Décembrettes 7. Munich : Lincom Europa.185-210.

NAMER Fiammetta \& HATHOUT Nabil. 2020. « ParaDis and Démonette. From Theory to Resources for Derivational Paradigms ». The Prague Bulletin of Mathematical Linguistics 114 (1). 5-34.

PHARIES David. 2002. Diccionario etimológico de los sufijos españoles y de otros elementos finales. Madrid : Gredos.

PICOCHE Jacqueline. 1992. Précis de lexicologie française. Paris : Nathan.

PLAG Ingo. 2003. Word-formation in English. Cambridge : Cambridge University Press.

POHL Jacques. 1970. « Remarques sur les antonymes ». In Cohen D., Mélanges Marcel Cohen. La

Haye/Paris : Mouton, 185-192.

RAINER Franz. 2018. «Word formation and word history: the case of CAPITALIST and CAPITALISM ». In Bonami O. et al., The lexeme in descriptive and theoretical morphology. Berlin : Language Science Press. 43-65.

REY-DEBOVE Josette. 1971. Étude linguistique et sémiotique des dictionnaires français contemporains. La Haye : Mouton

ROCHÉ Michel. 2007. « Logique lexicale et morphologique : la dérivation en -isme ». In Montermini F., Boyé G., \& Hathout N., Selected Proceedings of the 5th Décembrettes: Morphology in Toulouse. Somerville : Cascadilla Proceedings Project. 45-58.

ROCHÉ Michel. 2008. «Structuration du lexique et principe d'économie : le cas des ethniques ». In Durand J., Habert B. \& Laks B., Congrès Mondial de Linguistique Française-CMLF'08. Paris : Institut de Linguistique Française. 1571-1585.

ROCHÉ Michel. 2010. « Base, thème, radical ». Recherches linguistiques de Vincennes 39. 95-134.

ROCHÉ Michel. 2011. «Quel traitement unifié pour les dérivations en -isme et en -iste ? ». In Roché M. et al., Des unités morphologiques au lexique. Paris : Lavoisier. 69-143.

SANTIAGO LACUESTA Ramon \& BUSTOS GISBERT Eugenio. 1999. « La derivación nominal ». In Bosque I. \& Demonte V., Gramática descriptiva de la lengua española (vol.3). Madrid : Espasa Calpe. 4505-4594.

SEARLE John. 1982 (1979). Sens et expression. Paris : Minuit (trad. Joëlle Proust). 
STRNADOVÁ Jana. 2014. Les réseaux adjectivaux. Sur la grammaire des adjectifs dénominaux en français.

Thèse de doctorat. Université Paris 7 Denis Diderot.

Trésor de la langue française informatisé

Le Petit Robert 2017 électronique

\section{NOTES}

1. Cet article précise Agabalian (2019a) et améliore et révise certains points d'Agabalian (2019b).

2. Cet article est le fruit d'une réflexion entamée en 2017.

3. L'exclusion d'environ 130 ISMES a toutefois été problématique d'après ces critères. Pour plus de détails voir Agabalian (2019b).

4. Pour d'autres d'analyses voir aussi pour le français: Dubois (1962a, 1962b) ; Corbin (1988); Dubois \& Dubois-Charlier (1999). Pour le suffixe anglais -ism: Marchand (1960); Plag (2003); Baeskow (2004). Pour le suffixe espagnol -ismo : Beniers Jacobs (1992) ; Santiago Lacuesta \& Bustos Gisbert (1999) ; Muñoz Armijo (2010).

5. textometrie.ens-lyon.fr

6. Croyance est ambigu car il peut, comme attachement, instancier la variable <valorisation $>$ mais il a la particularité de pouvoir constituer un hyperonyme pour certains ISMES.

7. La variation sémantique chez les ISMEs valorisationnels fera l'objet d'une publication ultérieure.

8. Voir Chantraine (1979: 141-144), Pharies (2002: 356, 358), Roché (2011:120).

9. La distinction entre ces deux types de valorisations est présentée plus en détail dans Agabalian (2019a).

10. Le lecteur sera peut-être surpris de découvrir que stylisme possède aussi ce sens, comme il a peut-être été surpris de découvrir un autre sens pour naturisme. Nous rappelons que nous ne traiterons pas les questions de polysémie et d'homonymie dans cet article.

11. Lorsque la <valorisation> n'est pas un acte d'énonciation alors que le <résultat> est une idéalité (par exemple <résultat : doctrine> qui <valorisation : fait prévaloir > ...), la valorisation est de type modal. Cette configuration est rare dans le corpus.

12. Pour nous, la base d'un lexème dérivé n'est pas nécessairement un lexème. Elle peut aussi être un syntagme ou appartenir à une catégorie non lexicale (comme un pronom par exemple).

13. Nous simplifions ' $<$ valorisation $>$ de $<x$ : quelque chose $>$ par $<y>$ ' par la relative périphrastique ' $<\mathrm{x}$ : ce que $>\mathrm{y}><$ valorise' mais les deux structures ne sont pas équivalentes. La relative périphrastique peut participer à une relation attributive aussi bien avec le <résultat> (Ce que le végane valorise est un mode de vie par exemple) qu'avec l'objet valorisé (Ce que le végane valorise est la non-exploitation des animaux par exemple).

14. Ce phénomène (syncrétisme) est aussi dans Roché (2008) ou dans Namer (2013) pour la formation des verbes en -is(er).

15. Pour toute question sur la formation ou sur des cas particuliers voir Roché (2011). Voir aussi les auteurs cités dans la note 4 .

16. Le modèle canonique veut qu'une relation de dérivation soit décrite 1) comme une relation unidirectionnelle (donc pas ' $\longleftrightarrow$ '), 2) unissant seulement deux unités (donc pas de formation triangulaire par exemple) et 3) allant toujours de l'unité la moins complexe à la plus complexe (donc pas de rétroformation).

17. Pour la remise en question du modèle canonique et/ou une défense de la morphologie paradigmatique, voir notamment Bauer (1997), Hathout \& Namer (2014), Strnadová (2014), Lignon, Namer \& Villoing (2014), Bonami \& Strnadová (2019), Namer \& Hathout (2020). 
18. " Thème » au sens de Bonami \& Boyé (2003) étendu à la morphologie constructionnelle.

19. Voir des exemples d'applications chez Amiot \& Dal (2007) ou chez Roché (2010).

20. D'après le TLFi, hédonisme a été formé en français. Il n'a pas été emprunté au grec.

21. Pour ce qui est du rôle déterminant du sens pour décrire les préfixations, nous renvoyons à Corbin (1999) et à Iacobini \& Scalise (2008).

22. Pour les NPan, a été oublié victorianisme (non valorisationnel) dans lequel on retrouve le nom de la reine Victoria mais la base est plutôt l'adjectif victorien. Pour les noms communs, ont été oubliés sibyllisme (sibylle comme objet valorisé) et guidisme (guide comme agent de valorisation)

23. Les exceptions sont quelques ISMEs à valorisation attitudinale que les dictionnaires définissent comme des affects positifs envers <b>, par exemple africanisme: "amour de l'Afrique " (TLFi). Défini de cette manière, on ne voit pas pourquoi une rivalité entre l'Afrique et une autre partie du monde serait nécessaire pour qu'africanisme puisse signifier ce qu'il signifie. Toutefois, il est possible que les quelques cas de ce genre soient contingents, dus à des aléas de formulation de la définition. De plus nous, il n'a pas toujours été possible de connaître le contexte de première attestation des ISMES à valorisation attitudinale car la connaissance de ce contexte peut parfois indiquer qu'il a existé une rivalité à l'origine.

24. Sur cette question de la distinction voir la tribune "Misère du viandisme » (Dalibor Frioux) dans Libération, 10 avril 2016.

25. Cette opposition ne fonctionne pas pour les ISMEs à valorisation attitudinale. Les carriéristes ou les égoïstes, par exemple, ne sont pas les partisans d'une cause ou d'une vérité (comme le sont pas, par exemple, les véganes ou les existentialistes).

26. Nous regroupons sous le terme « inversion » toutes les relations où l'opposition ne repose pas sur un opérateur de négation mais d'inversion : les converses (ou réciproques) du type maître/ serviteur, mari/femme, vendre/acheter (Lyons, 1978 : 226-227 ; Lyons, 1970 : 357-358 ; Cruse, 1986 : 231-232 ; Picoche, 1992: 101), les réversifs du type remplir/vider, habiller/déshabiller, tuer/ ressusciter (Lyons, 1978 : 227-228 ; Cruse, 1986 : 226-228) et les antipodaux du type hiver/été, tête/ pied, noir/blanc (Lyons, $1978: 229$; Cruse, $1986: 224-225$ ).

27. Peuple est très polysémique. Dans populisme, il n'est jamais sélectionné avec son sens juridique ('ensemble d'humains de même nationalité'). Il est toujours sélectionné avec son sens sociologique ('ensemble d'humains regroupant les classes défavorisées d'un pays') auquel s'ajoute, parfois, son sens ethnique ('ensemble d'humains ethniquement homogènes au sein d'un pays'). Quant à son sens politique ('ensemble d'humains constituant le souverain d'une démocratie'), il est sélectionné par les gens accusés de populisme pour réhabiliter le terme.

28. Au sens classique du terme: une relation entre deux prédicats qui ne peuvent ni être vrais tous les deux en même temps ni être faux tous les deux en même temps.

29. On peut aussi considérer qu'il s'agit d'une construction appellative mais nous préférons utiliser le concept de dénomination.

30. L-E représente tout locuteur qui est aussi énonciateur (en particulier les adeptes de la doctrine dénommés en -iste ou autrement) et $\mathrm{E}$ représente tout énonciateur qui n'est pas un locuteur (par exemple la loi, la tradition, la morale...).

31. Dans Agabalian (2019a) nous défendons le fait que les ISMEs à valorisation modale sont des noms de discours.

32. Nous nous inspirons d'une représentation schématique trouvée chez Éliade \& Couliano (2016: 122) pour les doctrines christologiques.

33. Le lexème égalitarisme connaît des emplois péjoratifs (notamment à cause d'une valorisation excessive de l'égalité) mais la valorisation d'une égalité de traitement de tous les hommes (en droit ou en économie) est bien attestée dans les encyclopédies.

34. La double flèche verticale représente la hiérarchisation. Les symboles + et - indiquent respectivement ce qui est supériorisé (forcément $<\mathrm{b}>$ ) et infériorisé (forcément $<\mathrm{d}>$ ). 


\section{RÉSUMÉS}

Cet article est un ensemble de propositions pour une description sémantique formalisée des noms de doctrines, de théories, de mouvements, de religions en -isme ainsi que de certains noms d'attitudes en -isme. Le sens construit de ces noms consiste, la plupart du temps, en une valorisation de leur base : par exemple 'valorisation de l'autonomie' pour autonomisme (Roché, 2011). Dans la première section, nous proposons de décrire le sens construit de ces noms comme un ensemble de variables à instancier et nous exposons les écarts entre sens construit et sens attestés. Dans la seconde section, nous proposons d'intégrer à cette description le fait que l'interprétation valorisationnelle de ces noms est toujours opérée dans un contexte de rivalité.

This article is a set of proposals for a formal semantic description of nouns of doctrines, theories, movements, religions in -isme and some nouns of attitudes in -isme. The constructed meaning of these nouns consists often in a valorization of their base, for instance 'valorization of autonomy' for autonomisme (Roché, 2011). In the first section, I will propose to describe the constructed meaning of those nouns as a set of variables to be instantiated and I show the discrepancies between the constructed meaning and the attested meanings. In the second section, I propose to integrate in that description the fact that the valorizational interpretation is operated in a context of rivalry.

\section{INDEX}

Mots-clés : suffixe -isme, doctrine, attitude, idéologie, valorisation

Keywords : suffix -ism, doctrine, attitude, ideology, valorization

\section{AUTEUR \\ GRIGORY AGABALIAN}

Université de Paris - Philépol EA 7538 